\title{
X-ray Reflectivity Measurements and Landau Theory of Smectic Wetting in Liquid Crystal-Benzyl Alcohol Mixtures
}

\section{Citation}

Kellogg, G. J., Peter S. Pershan, E. H. Kawamoto, W. Foster, Moshe Deutsch, and B. M. Ocko. 1995. X-ray reflectivity measurements and Landau theory of smectic wetting in liquid crystalbenzyl alcohol mixtures. Physical Review E 51(5): 4709-4726.

\section{Published Version}

doi:10.1103/PhysRevE.51.4709

\section{Permanent link}

http://nrs.harvard.edu/urn-3:HUL.InstRepos:10357484

\section{Terms of Use}

This article was downloaded from Harvard University's DASH repository, and is made available under the terms and conditions applicable to Other Posted Material, as set forth at http:// nrs.harvard.edu/urn-3:HUL.InstRepos:dash.current.terms-of-use\#LAA

\section{Share Your Story}

The Harvard community has made this article openly available.

Please share how this access benefits you. Submit a story.

\section{Accessibility}




\title{
X-ray reflectivity measurements and Landau theory of smectic wetting in liquid crystal-benzyl alcohol mixtures
}

\author{
G.J. Kellogg, ${ }^{*}$ P.S. Pershan, E.H. Kawamoto, and W. F. Foster \\ Department of Physics and Division of Applied Sciences, Harvard University, Cambridge, Massachusetts 02138 \\ Moshe Deutsch \\ Physics Department, Bar-Ilan University, Ramat-Gan 52100, Israel \\ B.M. Ocko \\ Department of Physics, Brookhaven National Laboratory, Upton, New York 11973
}

(Received 17 October 1994)

\begin{abstract}
Smectic layering has been observed at the free surface of decylcyanobiphenyl (10CB) and dodecylcyanobiphenyl (12CB) and mixtures of $10 \mathrm{CB}$ and $12 \mathrm{CB}$ with benzyl alcohol (BA). The effect of $\mathrm{BA}$ is to suppress the bulk isotropic-smectic transition temperature $T_{I A}$ and surface layer "transition" temperatures $T_{j}$, as well as sharpening these surface transitions by reducing the temperature range $\Delta T$ over which layers grow. The observed sharpening appears to saturate for concentrations $x \geq 0.118$. A Landau theory for the growth of a single layer $j$ has been developed, in which the free energy contains a term coupling the concentration $x$ to the local smectic order parameter $\psi_{j}$ such that $F_{j} \sim x \psi_{j}^{2}$. Applying this theory to pure 12CB and eight mixtures of 12CB-BA, we find that the $j=1 \rightarrow j=2$ transition is continuous in pure $12 \mathrm{CB}$, but that the addition of small amounts of impurity drives the transition first order.
\end{abstract}

PACS number(s): 68.10.-m, 78.70.Ck

\section{INTRODUCTION}

One of the great successes of x-ray specular reflectivity (XSR) has been the observation of surface smectic layering in liquid crystals [1-8]. In smectic layering, roughly sinusoidal mass and electron-density oscillations develop normal to the interface between the nematic $(N)$ or isotropic $(I)$ liquid crystal and the vapor or a solid substrate. The availability of intense $\mathrm{x}$-ray sources has allowed XSR measurements to be carried out to large momentum transfers $q_{z}$, obtaining information on correspondingly small spatial length scales $\Delta z \approx 2 \pi / q_{z}$. Although optical reflectivity techniques, such as ellipsometry and Brewster angle microscopy, are sensitive to surface phases, they cannot directly resolve structure on the molecular length scale. For our purposes, applicability of a kinematic approximation also gives XSR some advantage over other techniques, such as forward-recoil electron spectroscopy and x-ray photoelectron spectroscopy, which in principle have comparable spatial resolution, but which do not directly measure the electron distribution $\rho_{e}(z)$. Offsetting the sensitivity to the electron density profile $\rho_{e}(z)$ obtained from XSR is the fact that the measured reflectivity $R\left(q_{z}\right)$ is an intensity, and does not contain phase information; this leads to the well-known problem of nonuniqueness of $\rho_{e}(z)$ for a given $R\left(q_{z}\right)$ [9].

\footnotetext{
${ }^{*}$ Present address: Department of Materials Science and Engineering, Massachusetts Institute of Technology, Cambridge, MA 02139.
}

In many cases, physical intuition or other information about the structure is sufficient to determine the most reasonable profile. We will also argue below that, for the current measurements, the exact details of the profile are not important, and that relative changes in the structure can be believed with a high level of confidence.

A significant reason for interest in smectic layering is that it is an example of wetting. In this case, the lowersymmetry smectic- $A$ phase appears at an interface of the higher-symmetry $I$ or $N$ phase with a substrate, or at the free surface, as bulk coexistence between phases is approached. The smectic- $A$ phase is characterized by the smectic order parameter $\psi=|\psi| e^{i \phi}$, which measures the amplitude $(|\psi|)$ and phase $(\phi)$ of electron-density oscillations of period $d$ along the direction $z$ :

$$
\rho_{e}(\mathbf{r})=\overline{\rho_{e}}+\operatorname{Re}\left[\psi e^{i(2 \pi / d) z}\right]
$$

The cyanobiphenyl liquid crystals $(n \mathrm{CB})$ exhibit a variety of bulk liquid crystalline phases. These homologs consist of two phenyl rings with a cyano end group on one end and an alkane tail $\mathrm{C}_{n} \mathrm{H}_{2 n+1}$ on the other; hence the " $n$ " refers to the tail length. For short tail length $(n \leq 7)$, when temperature is lowered at constant pressure, the $I$ phase undergoes a first-order transition to the $N$ phase and, on further cooling, the nematic phase crystallizes; there is no smectic phase. For intermediate chain lengths $(8 \leq n<9)$, an additional $N-A$ transition occurs which is either second order or weakly first order. One recent experiment on the cyanobiphenyls suggests that the $N-A$ transition is always weakly first order [10], even for materials with unmeasurably small latent heats, such as $8 \mathrm{CB}[11]$. (A recent study of cyanobiphenyls sug- 
gests that the $N-A$ transition is always weakly first order [10], in contrast to the results of a number of heat capacity and $\mathrm{x}$-ray scattering experiments indicating that the $N-A$ transition is second order sufficiently far from the $I-N-A$ triple point $[11-13])$. The $N-A$ transition is clearly first-order for $n>9$, while the $N$ phase disappears entirely in $9 \mathrm{CB}-10 \mathrm{CB}$ mixtures with mole fraction $x \approx 0.310 \mathrm{CB}$; for $n>9.3$, there are only first-order $I-A$ and $A$-crystal transitions $[14,11,15-18]$.

The surface smectic layering of the $n \mathrm{CB}$ series was previously shown to exhibit an analogous dependence on $n$ as the bulk $N$ - $A$ transition temperature $T_{N A}$ is approached from above $[3,7]$. This layering is evident in the behavior of a spatially dependent smectic order parameter $\psi(z)$, where $z$ is measured relative to the interface. For shorter-chain molecules (8CB and 9CB), the modeled $\psi(z)$ grows continuously to saturation as reduced temperature $t=\left(T-T_{N A}\right) / T_{N A} \rightarrow 0$, with an exponential penetration $\psi(z) \sim e^{-z / \xi_{\|}(t)}$, where $\xi_{\|} \sim t^{-\nu_{\|}}$, the bulk correlation length measured along the director, i.e., normal to the layers. The divergence of $\xi_{\|}$in a continuous manner suggests that smectic wetting for these systems is analogous to critical adsorption [19]. For materials with first-order $N-A$ (mixtures of $9 \mathrm{CB}$ and 10CB with mole fraction of $9 \mathrm{CB} x \leq 0.3)$ or $I-A(10 \mathrm{CB}, 11 \mathrm{CB}$, and $12 \mathrm{CB}$ ) transitions, the evolution of $\psi(z)$ with $t$ becomes progressively more discontinuous, and it appears that the surface is wetted by a finite number of layers before the bulk transition. However, the distinction between continuous and discrete layering could not be unambiguously made in even the longest-chain $n$ CB: the observed width $\Delta t\left[t=\left(T-T_{I A}\right) / T_{I A}\right]$ of layering transitions in 12CB was apparently nonzero for all measured transitions.

Though we use the term "transitions" to describe the growth of single smectic layers, it is important to recognize that this growth may not be a true phase transition. For example, the growth of smectic order as temperature is decreased could be dominated by the nonlinear temperature dependence of a layering susceptibility in the presence of either a real or effective surface field. Even if the system had a tendency towards a second-order transition, these fields would quench critical fluctuations and formally suppress any real phase transition. This is similar to the effects of external fields on bulk second-order phase transitions between phases of the same symmetry [20]. We note, however, that the formation of a smectic layer at the surface can occur via a first-order transition since the presence of an external field does not suppress discontinuities in the order parameter; however, it can give rise to prominent pre- and post-transitional effects on the order parameter. In any case, the term "transition" will be used here as shorthand for the growth of a single smectic layer.

The initial XSR experiments on liquid crystal surfaces that were cited above stimulated the development of a number of approaches to understanding smectic wetting in the mean field approximation. While bulk secondorder transitions between the $N$ and $A$ phases are dominated by fluctuations, making mean field theory inapplicable, it should be useful in gaining a qualitative understanding of smectic wetting. For long-chain materials
( $n \geq 9$ ), the bulk $N$ - $A$ or $I$ - $A$ transitions are first order, so that the universal behavior associated with critical phenomena is absent and analytic theories (such as the renormalization group) are inapplicable. Although fluctuation effects may still be important, and although it may be necessary to include them in numerical calculations for either, or both, bulk and surface transitions, the presence of a free surface characterized by surface tension $\gamma$ does reduce the magnitude of fluctuations at the surface [21].

The first attempt to understand smectic layering consisted of a lattice model using Ising spins to represent smectic and nematic order. The calculation predicted the continuous growth of up to two surface smectic layers at the free interface of the $I$ phase [22]. A density-functional theory [21], using an anisotropic potential similar to that of the original McMillan theory for bulk transitions to the $A$ phase [23], predicted two discontinuous layering transitions for the $I$ phase. A similar density-functional calculation, with additional translational-rotational terms added to the single-particle potential, indicated the continuous growth of 3-4 smectic layers [24]. Recently, the same interparticle interactions were used to study layering in an isotropic fluid at an interface characterized by a long-range potential $\left(\sim 1 / z^{3}, z \equiv\right.$ distance from the interface) [25]. While this calculation is more appropriate for the problem of layering at a solid substrate, an interesting prediction is made in regard to the surface phase diagram. For a particular choice of relative values of surface and bulk interaction parameters, a region of incomplete wetting is found, with a finite number (in this case, 2) of first-order layering transitions terminated by critical points. For other choices of interaction parameters, this incomplete wetting goes over to complete wetting, as expressed by an infinite number of layering transitions. This model does not treat layering of the $N$ phase. None of the approaches discussed here has produced layering in the $N$ phase which is continuous for short chains evolving to first-order transitions in the smectic- $A$ phase, as was inferred from the experimental results, although the recent calculations do suggest that the progression from short to long chains can induce a progression from continuous to first-order layering [25].

In an effort to determine whether layers grow continuously or with a discrete jump in the local smectic order parameter of layer $j, \psi_{j}$, XSR data for the temperature region surrounding the growth of a single layer, $j=2$, have been modeled by electron-density profiles whose parameters are a measure of the amplitude of the electron-density oscillation of the surface smectic layers. Two materials were studied with direct $I$ - $A$ transitions, $10 \mathrm{CB}$ and $12 \mathrm{CB}$, as well as mixtures of these liquid crystals with benzyl alcohol, $\mathrm{C}_{7} \mathrm{H}_{8} \mathrm{O}$ (BA). It is well known that, although the presence of small amounts of impurity does not change the topology of a thermal equilibrium phase diagram, it can both shift the phase boundaries and change the order of transitions [20, 26]. By comparing the systematic behavior of mixtures as a function of solute concentration it is possible to gain greater insight into the behavior of the pure materials. By way of illustration, it has been shown that small amounts of im- 
purity can dramatically alter the wetting properties of binary liquids $[27,28]$. The measurements we report below are a demonstration of the qualitative and quantitative significance of impurities in the smectic wetting of liquid crystals, and in particular suggest that the $j=1 \rightarrow j=2$ transition in pure $12 \mathrm{CB}$ is continuous, but is driven first order by small concentrations of BA.

\section{REFLECTIVITY THEORY AND ELECTRON-DENSITY MODEL}

The principles of x-ray specular reflectivity have been described in a number of publications $[29,1,7]$, and we present here the important relationships without development. The primary results of interest are the Born approximation expression for the reflectivity due to structure normal to the interface and the effects of surface roughness, here due to thermally excited capillary waves.

For an infinitely sharp interface between vacuum or air and homogeneous matter, unpolarized $\mathrm{x}$ rays are specularly reflected with an intensity described by the usual Fresnel form familiar from classical electromagnetic wave theory $[29,7]$,

$$
R_{F}\left(q_{z}\right) \approx\left|\frac{q_{z}-\left(q_{z}^{2}-q_{c}^{2}\right)^{\frac{1}{2}}}{q_{z}+\left(q_{z}^{2}-q_{c}^{2}\right)^{\frac{1}{2}}}\right|^{2}, \quad q_{z}>q_{c}
$$

Here, $q_{z}$ is the momentum transfer normal to the interface and $q_{c} \approx 4 \sqrt{\overline{\rho_{e}} r_{0} \pi}$ is the critical wave vector, such that $R_{F}\left(q_{z} \leq q_{c}\right)=1$. With an average bulk electron density of $\overline{\rho_{e}}=3.2 \times 10^{23} \mathrm{~cm}^{-3}$ (typical of hydrocarbons) and $r_{0}=2.818 \times 10^{-13} \mathrm{~cm}, q_{c}=0.021 \AA^{-1}$.

Real surfaces have structure, both normal to and within the plane defining them. Both cases can be treated within the Born approximation if the angle of incidence is large compared to the critical angle so that refraction effects can be neglected and the scattering is weak. If one expresses the structure in the $z$ direction averaged over the coherence length of the $\mathrm{x}$ rays within the plane as $\rho_{e}(z)$, the reflectivity is given by

$$
R\left(q_{z}\right)=\left|\Phi\left(q_{z}\right)\right|^{2} R_{F}\left(q_{z}\right)
$$

with

$$
\Phi\left(q_{z}\right)=\frac{1}{\overline{\rho_{e}}} \int_{-\infty}^{\infty} d z e^{-i q_{z} z} \frac{\partial \rho_{e}(z)}{\partial z} .
$$

For the case of a fluctuating or rough interface, additional scattering arises at $q_{z}, \mathbf{q}_{\perp} \neq \mathbf{0}$, where $\mathbf{q}_{\perp}$ lies within the plane of the surface. This diffuse scattering will not be discussed in detail here. It is clear, however, that diffuse scattering will be accompanied by decrease in the magnitude of the specularly reflected radiation relative to that from a sharp interface. It can be shown that the effect of diffuse scattering by thermal capillary waves, which are present at all liquid-vapor interfaces, decreases the specular signal in a manner reminiscent of the Debye-Waller factor in bulk diffraction which arises from thermal fluctuations [30, 31]:

$$
\frac{R}{R_{F}}=\left|\Phi\left(q_{z}\right)\right|^{2} e^{-q_{z}^{2} \sigma_{C}^{2}}
$$

where $\sigma_{C}$ is the root-mean-square roughness due to capillary waves. The value of $\sigma_{C}$ is determined by the temperature, surface tension, and the long-wavelength $q_{\min }$ and short-wavelength $q_{\max }$ cutoffs in the capillary wave spectrum $[32,33]$. It has been observed that although $q_{\min }$ is determined by the projection of the reflectometer resolution on the plane of the surface, in all practical experiments the reflectivity can be modeled by implicitly treating the interfacial widths of the model as a combination of the intrinsics widths (e.g., the inherent width $\sigma_{0}$ of the liquid-vapor interface) and the capillary wave roughness [34]. By modeling reflectivities from liquid surfaces in this way, it has been found that a consistent and physically meaningful choice for the short-wavelength capillary wave cutoff is $q_{\max } \approx \pi / a$, where $a$ is the molecular diameter.

To represent smectic layers at the surface of an isotropic liquid crystal, a model electron-density profile is needed that can capture the nearly sinusoidal oscillations of bulk smectic- $A$ liquid crystals while at the same time allowing the smectic order parameter $\psi_{j}$ of the $j$ th layer to vary as a function of temperature. This is a generalization of the sinusoidal model previously employed, which consisted of a sine-wave oscillation about the bulk electron density $\overline{\rho_{e}}$ penetrating a fixed distance into the bulk [3]. This ability to model varying amplitudes is especially important in regions where the transition $j \rightarrow j+1$ layers occurs: if these transitions are continuous, one must be able to model smectic order for the $j+1$ layer that is intermediate between the $I$ phase $\left(\psi_{j+1}=0\right)$ and a saturation level.

The model consists of an error-function interface, with electron density rising from zero to $\overline{\rho_{e}}$, representing the transition from the vapor to the liquid. To this are added oscillatory layers with zero net integrated electron density. Each layer $j$ consists of a central, positive Gaussian of amplitude $\overline{\rho_{e}} A_{j}$ at position $z_{j}=z_{0}+(j-1) d$, with two negative Gaussians of amplitude $-\frac{1}{2} \overline{\rho_{e}} A_{j}$ at positions $z_{j} \pm d / 2$; here, $z_{0}$ is an offset from the surface defined by the error function and $d$ is the smectic layer spacing. Bulk diffraction studies on $n \mathrm{CB}$ 's are consistent with a smectic- $A$ structure where the molecules pair head to head to form a bilayer which is the repeat unit of the one-dimensional density oscillation [35]. The positive Gaussian in the model can be thought of as representing the overlapping molecular cores of the bilayer and the negative Gaussians the lower density tails. Explicitly,

$$
\begin{aligned}
\rho_{e}(z)= & \frac{1}{2} \overline{\rho_{e}}\left[\operatorname{erf}\left(z / \sqrt{2} \sigma_{s}\right)+1\right] \\
& +\overline{\rho_{e}} \sum_{j=1}^{j=n} A_{j}\left[-\frac{1}{2} e^{-\frac{1}{2}\left(z_{j}+\frac{1}{2} d\right)^{2} / \sigma_{l}^{2}}+e^{-\frac{1}{2} z_{j}^{2} / \sigma_{l}^{2}}\right. \\
& \left.-\frac{1}{2} e^{-\frac{1}{2}\left(z_{j}-\frac{1}{2} d\right)^{2} / \sigma_{l}^{2}}\right]
\end{aligned}
$$

where $\sigma_{s}$ is the Gaussian width of the surface profile, made up of both surface roughness and intrinsic width, and $\sigma_{l}$ represents a similar quantity for the individual layers. 
It is important to realize that the exact nature of the model is not of great significance in the current study. The primary requirement is the ability to describe a single layer's change in smectic amplitude as a function of temperature; even if the independent-layer model does not represent the exact quantitative features of the structure, the relative changes in smectic order are well represented. This can be seen in that good fits can be made using the model where the only parameters with significant temperature dependence are the $A_{j}$. For this reason we use the $A_{j}$ to represent the layer smectic order parameter $\psi_{j}$.

\section{EXPERIMENTAL DETAILS}

\section{A. Sample cells and sample preparation}

To minimize temperature gradients across the samples which could smear transitions, the aluminum cells shown in Fig. 1 were built. These cells were made with the smallest x-ray windows which could safely accommodate the specular beam at all angles, measuring $9.5 \mathrm{~mm} \times 10 \mathrm{~mm}$; the windows themselves were made of $0.25 \mathrm{~mm}$ thick beryllium foil (to increase thermal conductivity), sealed to the body of the cell with zero-volume indium $\mathrm{O}$ rings. The cells were also designed to have a very small free volume, with a space of approximately $6 \mathrm{~mm}$ above the surface of the liquid. The liquid crystals themselves form a large puddle $0.1-0.3 \mathrm{~mm}$ thick on top of a $7.6 \mathrm{~cm}$ diam $\times 3.2 \mathrm{~mm}$ thick polished silicon wafer. Silicon was used rather than glass because its thermal conductivity $\left(K_{T} \approx 1.7 \mathrm{~W} \mathrm{~cm}^{-1} \mathrm{~K}^{-1}\right)$ is more than two orders of magnitude greater than that of glass $\left(K_{T} \approx 9 \times 10^{-3} \mathrm{~W} \mathrm{~cm}^{-1} \mathrm{~K}^{-1}\right)$.

These cells formed the inner stage of a two-stage temperature-controlled oven. The outer can consisted of a brass cylinder with $3.2 \mathrm{~mm}$ walls and a brass lid. Small kapton windows insured a dead space between the outer can and the inner cell. Temperature control was maintained to $\pm 0.5 \mathrm{mK}$ at the sample, with a YSI Model 72 temperature controller on the outer can and a custombuilt temperature controller for the inner cell; YSI 44011 thermistors were used for both control and temperature

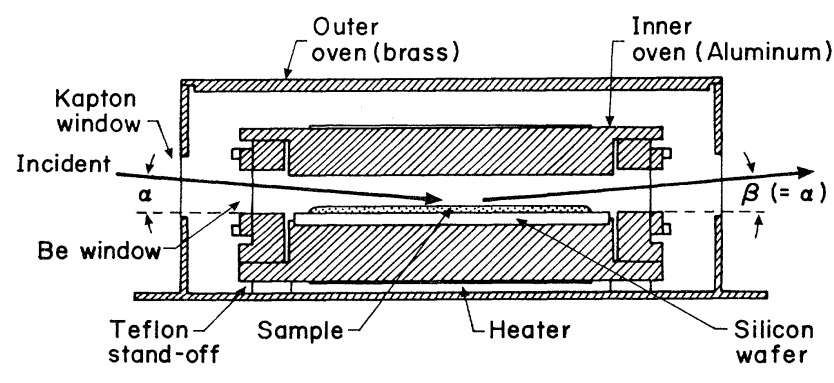

FIG. 1. Sample cell used for 12CB and 12CB-BA experiments; details of the construction are described in the text. Also shown is the path of the $x$-ray beam specularly reflected and grazing angles of incidence $\alpha$ and reflection $\beta$. measurement. The temperature gradient was found to be $|\Delta T| \leq 5 \mathrm{mK}$ between the edge of the sample wafer and the center of the cell.

In all cases, the silicon substrate was precleaned in a $\mathrm{H}_{2} \mathrm{SO}_{4}-\mathrm{H}_{2} \mathrm{O}_{2}$ bath and rinsed with deionized, purified water. After blowing the disk dry with dry nitrogen, it was placed in the inner cell. For the experiments using pure liquid crystals, they were added directly from the bottle without further purification.

Baker Analyzed benzyl alcohol $\left(\mathrm{C}_{7} \mathrm{H}_{8} \mathrm{O}\right)$ (BA) was used in the mixture experiments. In these experiments, BA was added to a known weight of liquid crystal using a microliter syringe; the sealed mixing bottle was heated to melt the sample, which was then placed in an ultrasonic water bath for $5 \mathrm{~min}$. The mixtures were then added to the hot silicon wafer in the liquid state; if any evidence of phase separation was observed, the sample was removed. We found through the course of these experiments that phase-separated samples could not be restored to a homogeneous state, even with heating as high as $80^{\circ} \mathrm{C}$. This is not surprising, considering the small diffusion constants of solutes in liquid crystals [36] and the thinness of the puddles.

Finally, it should be noted that the thinness of the liquid puddles acts to cutoff low-frequency, long-wavelength gravity waves caused by room vibrations. The macroscopic flatness (or low figure error) of these samples is demonstrated by the ability to measure a specular signal to very high angles.

\section{B. X-ray measurements}

Extensive use of both the Harvard rotating anode $\mathrm{x}$-ray source and beamline X22B at the National Synchrotron Light Source (NSLS) was made in acquiring the data. Both are equipped with special spectrometers built to allow scattering measurements from horizontal liquid surfaces and have been described in detail elsewhere [37, 7]. In terms of the kinematics of reflectivity, the spectrometers are identical. As the incident grazing angle $\alpha$ is increased by tilting down the x-ray beam reflected from a monochromator, the sample is lowered by an elevator; the specular beam is reflected at angle $\beta=\alpha$. Associated with $\alpha$ is a divergence $\Delta \alpha$ due to $\mathrm{x}$-ray slit sizes (and, at the synchrotron, the inherent divergence of the source). The size of the detector slit determines the detector resolution $\Delta \beta$. The outgoing slits were set to allow all of the specularly reflected $\mathrm{x}$-ray radiation to enter the detector; as explained elsewhere [5], this implies that for a flat surface the resolution $\Delta q_{z}$ is dominated by the incident resolution $\Delta \alpha$. For the Harvard and NSLS spectrometers these resolutions were $\Delta q_{z} \approx 4 \pi \Delta \alpha / \lambda \leq 2.6 \times 10^{-4} \AA^{-1}$.

All data on mixtures of $12 \mathrm{CB}$ with $\mathrm{BA}$ were taken at Harvard, with $\lambda=1.5405 \AA$. The pure $12 \mathrm{CB}$ data were taken at NSLS with a wavelength of $\lambda=1.5567 \AA$. Data on pure 10CB and 10CB-BA mixtures were taken at NSLS with $\lambda=1.5295 \AA$ and $\lambda=1.5374 \AA$.

A common background-subtraction procedure was used for all measurements. Because the intensity measured at the specular condition consists of the specular signal, stray background, and surface and bulk diffuse 
scattering which fall within the detector resolution, it is desirable to remove as much of the nonspecular scattering as possible. If the momentum transfer is defined as $\mathbf{q}=\left(q_{x}, q_{y}, q_{z}\right)$, with $q_{x}$ the momentum transfer within both the plane of the surface and the reflection plane and $q_{y}$ orthogonal to $q_{x}$ and $q_{z}$, then background subtraction should be performed at the smallest values of $q_{\perp}=\sqrt{q_{x}^{2}+q_{y}^{2}}$ which are practical. Unfortunately, for practical values of the reflectometer resolution, the fraction of the total diffuse scattering due to thermal capillary waves which lies under the specular peak is quite large. This is because capillary wave diffuse scattering is proportional to $1 / q_{\perp}^{2}$ so long as $q_{\perp} \lambda_{\text {surf }} \gg 1$, where $\lambda_{\text {surf }} \approx 2 \mathrm{~cm}$ is the capillary wavelength. Because significant amounts of diffuse scattering cannot be subtracted away from the specular signal, the background was taken as the average intensity of two points $\mathbf{q}=\left(q_{x}= \pm q_{z} / 300, q_{y}=0, q_{z}\right)$ well away from the specular peak, to avoid inadvertently subtracting any of the reflectivity signal. While this widely used procedure does not fully separate the diffuse scattering from the specular signal, in almost all cases it yields a reflectivity curve for which the effects of roughness can be well described by a Debye-Waller-like factor with an effective, $q_{z}$-independent value of $\sigma_{C}$.

\section{RESULTS}

Reflectivity measurements as a function of $q_{z}$ and $T, R\left(q_{z}, T\right)$, were performed on samples of pure $12 \mathrm{CB}$, pure 10CB, and mixtures of both materials with BA. As will be shown below, layer transitions as a function of temperature can be characterized by a combination of these measurements and a restricted set of temperature measurements, $R\left(q_{z}^{*}, T\right)$, at a fixed wave vector $q_{z}^{*} \approx 0.925 q_{0}\left(q_{0}=2 \pi / d\right)$; in terms of reduced temperature $t=\left(T-T_{I A}\right) / T_{I A}$, the latter measurements are denoted $R\left(q_{z}^{*}, t\right)$. The measurements $R\left(q_{z}^{*}, T\right)$ were used in two ways. First, constant rate transition temperature drifts of a few $\mathrm{mK} / \mathrm{h}$ are ubiquitous in liquid crystals; this drift was apparent in a shift $\Delta T$ in the temperature dependence of features in $R\left(q_{z}^{*}, T\right)$. The shift $\Delta T$ of a curve with respect to one measured at an earlier time was used to generate a corrected bulk transition temperature, $T_{I A} \rightarrow T_{I A}+\Delta T$. This in turn provides a corrected reduced temperature. When adjusted in this way, two curves with a temperature shift collapse almost identically onto one another. This gives us confidence temperature dependence of the surface structure remained consistent over the course of the measurement. By repeatedly measuring $R\left(q_{z}^{*}, T\right)$ we have corrected the reduced temperatures of full reflectivity measurements taken over the course of several hours to several days on a single sample. The second use of $R\left(q_{z}^{*}, T\right)$ was to generate $A_{2}$ values in the model of Eq. (6) at temperatures between those of full reflectivity measurements $R\left(q_{z}, T\right)$ through an interpolation process which will be described below.

The procedure followed for each sample was to measure $R\left(q_{z}^{*}, T\right)$ several times at the beginning of the exper- iment. When a constant drift had been achieved, specular reflectivities $R\left(q_{z}, T\right)$ were measured in the region of $j=1 \rightarrow j=2$. A measurement of $R\left(q_{z}^{*}, T\right)$ was performed before and after each full reflectivity. For all materials, $R\left(q_{z}^{*}, T\right)$ was measured by changing the temperature and allowing the reflected intensity to stabilize, assuring that at each temperature the surface structure had equilibrated. Both descending and ascending data were measured. No sample showed hysteresis, to within the $\pm 5 \mathrm{mK}$ afforded by the measurement.

In order to determine the reduced temperature $t$, the bulk transition temperature $T_{I A}$ was measured for each sample after all reflectivity measurements were performed. In each case, $T_{I A}$ was determined by translating the sample through the beam with fixed angles $\alpha \neq \beta$ such that $\mathbf{q}=\left(q_{0} / 300,0, q_{0}\right)$ while lowering the temperature in $5 \mathrm{mK}$ increments near the transition. This allowed for discrimination against the specular signal, both from the surface and from bulk domains oriented with layers parallel to the surface. With each change in temperature, at least $10 \mathrm{~min}$ were allowed for equilibration; constant intensity was also used as an indicator of equilibrium. A sudden increase in intensity (of more than two orders of magnitude) as well as the development of a non-resolution-limited peak (signaling smectic domains with a spread of layer normals) were taken as indicators of the transition to the bulk $A$ phase.

\section{A. 12CB and 12CB-BA mixtures}

For the pure $12 \mathrm{CB}$ sample, the bulk transition temperature was measured to be $T_{I A}=58.086 \pm 0.005^{\circ} \mathrm{C}$ $(331.236 \mathrm{~K})$. This is the value for a particular sample after a period of drift, and does not represent the value extrapolated back to the time of the introduction of the sample to the scattering cell.

Full specular reflectivities were measured at twelve reduced temperatures in the $j=1 \rightarrow j=2$ region. Figure 2 shows two normalized reflectivities $R / R_{F}$ and model fits, for temperatures just below and just above the feature in $R\left(q_{z}^{*}=0.15 \AA^{-1}, t\right)$ associated with the growth of the second layer. The increase in intensity at $q_{z}^{*}=0.15 \AA^{-1}$ in $R / R_{F}$ can be seen in the inset of Fig. 2 , where $R\left(0.15 \AA^{-1}, t\right)$ is displayed. The "steps" signal the localized addition of layers.

For these measurements, we have found that good fits to the data can be made using an electron density as expressed by Eq. (6). The behavior of the parameters is as follows

First, all 12CB, 12CB-BA, 10CB, and 10CB-BA reflectivities were fitted with the layer roughness fixed at $\sigma_{l}=5.0 \AA$. We found that we could vary this parameter from $\sigma_{l} \approx 0.5 \AA$ to $\sigma_{l} \approx 8.0 \AA$ without noticeable changes in the quality of the fits. We attribute this to the fact that the limited range in $q_{z}$ measured in these experiments was not sufficient to determine short-length-scale variations in $\rho_{e}(z)$.

For pure $12 \mathrm{CB}$, all parameters other than the layer amplitudes $A_{j}$ show very little temperature dependence, with the exception of the layer spacing $d$. An abrupt 
change from $d=36.74 \pm 0.14 \AA$ to $d=38.28 \pm 0.09 \AA$ occurs upon second layer formation. The reflectivity modeled by Eq. (6) is quite sensitive to $d$; the combination of thickness and offset $z_{0}$ determines the position of the interference minimum. The possibility that this change in apparent layer thickness was due to different thicknesses for the first and second layers was explored by allowing the two length scales to independently vary; the uncertainties associated with these fits were so large as to make the two lengths indistinguishable. Fixing the thickness at the average value of $\bar{d}=37.51 \AA$ resulted in poorer fits, with the goodness-of-fit parameter $\chi^{2}$ increasing by approximately a factor of 2 for data well above and well below the transition. We note that all fitted values are smaller than the bulk value of $d=39.15 \AA$.

The position of the center of the first bilayer, $z_{0}$, was found to be nearly constant, $\overline{z_{0}}=19.70 \pm 0.04 \AA$. Fits with constant $z_{0}$ yielded identical results to within the quoted errors as the fits allowing $z_{0}$ to float. This value is almost exactly $d / 2$ for the bulk, but is somewhat larger than $d / 2$ for the surface layers $\left(\overline{z_{0}}=0.515 \bar{d}\right.$ for $j=2$, $\overline{z_{0}}=0.525 \bar{d}$ for $j=1$ ).

The surface roughness $\sigma_{s}$ shows some systematic variation with $t$, from a high value of $5.05 \pm 0.05 \AA$ at low temperatures to a low value of $4.89 \pm 0.04 \AA$ at high

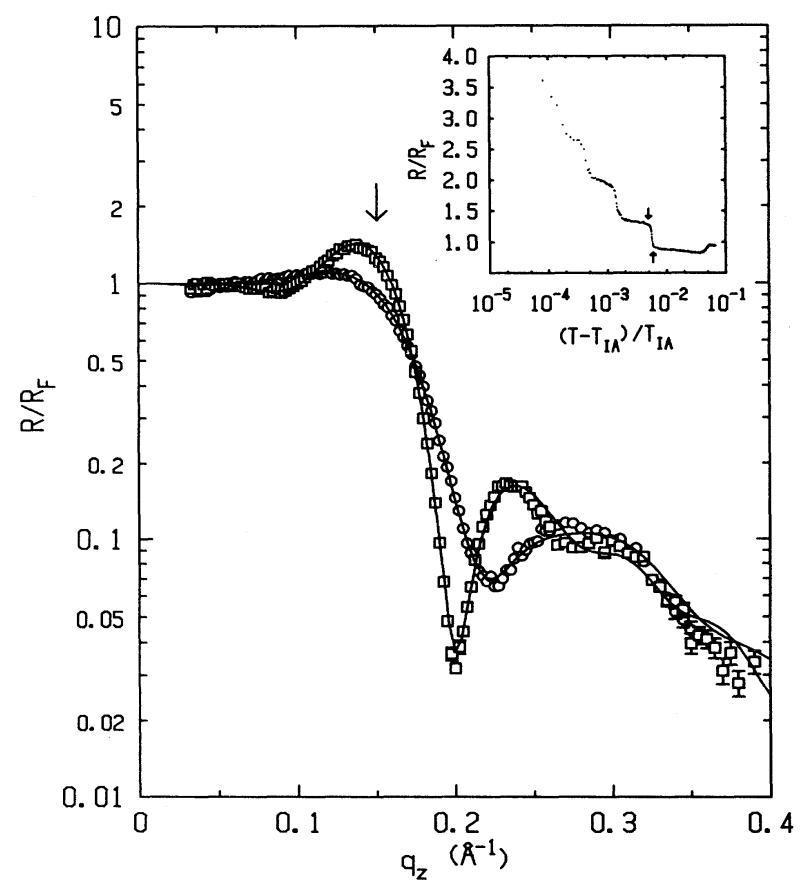

FIG. 2. X-ray specular reflectivity of pure $12 \mathrm{CB}$ at a reduced temperatures $t=5.98 \times 10^{-3}(\circ)$ and $t=4.96 \times 10^{-3}$ $(\square)$. The solid lines are fits to the independent-layer model discussed in the text. The inset shows $R\left(0.15 \AA^{-1}, t\right)$, taken at $q_{z}=0.15 \AA^{-1}$ marked by the arrows on the reflectivity plot. The temperatures of the reflectivities are shown as well in the inset, illustrating the "step" which corresponds to $j=1 \rightarrow j=2$. temperatures. Fixing the roughness at the average value resulted in an increase in $\chi^{2}$ of 10-20\% for low temperature and high temperature fits. Apparently, the growth of a second smectic layer does not significantly alter the microscopic roughness or intrinsic width of the interface.

Figure 3 shows the fitted layer amplitudes $A_{1}, A_{2}$, and $A_{3}$ as a function of reduced temperature for pure 12CB. (Also shown is $x_{0}=0.263 \mathrm{BA}$ in $12 \mathrm{CB}$, to be discussed below.) As expected, the second-layer amplitude $A_{2}$ undergoes the largest change with temperature, from $A_{2} \approx 0.022$ at the highest temperature to $A_{2} \approx 0.09$ at the lowest temperature. Amplitude $A_{1}$ is nearly constant, with average value $\overline{A_{1}}=0.127$. We attribute the scatter in $A_{1}$ to systematic errors and/or correlations between the fitting parameters. Fixing $A_{1}$ at the average value increases $\chi^{2}$ by $\leq 30 \%$. Finally, $A_{3}$ is nearly zero. Fixing $A_{3}=0$ results in an increase in $\chi^{2}$ of $\approx 50 \%$ for the low temperature data.

In addition to pure $12 \mathrm{CB}$, samples containing $\mathrm{BA}$ at eight different concentrations were measured. The concentrations (weight percentages) studied were $x_{0}=$ $0.031(1 \%), \quad x_{0}=0.076(2.5 \%), \quad x_{0}=0.090(3 \%), \quad x_{0}=$ $0.118(4 \%), \quad x_{0}=0.145(5 \%), \quad x_{0}=0.170(6 \%), \quad x_{0}=$ $0.207(7.5 \%)$, and $x_{0}=0.263(10 \%)$. Samples with larger concentrations of $\mathrm{BA}$ were not measured in detail be-

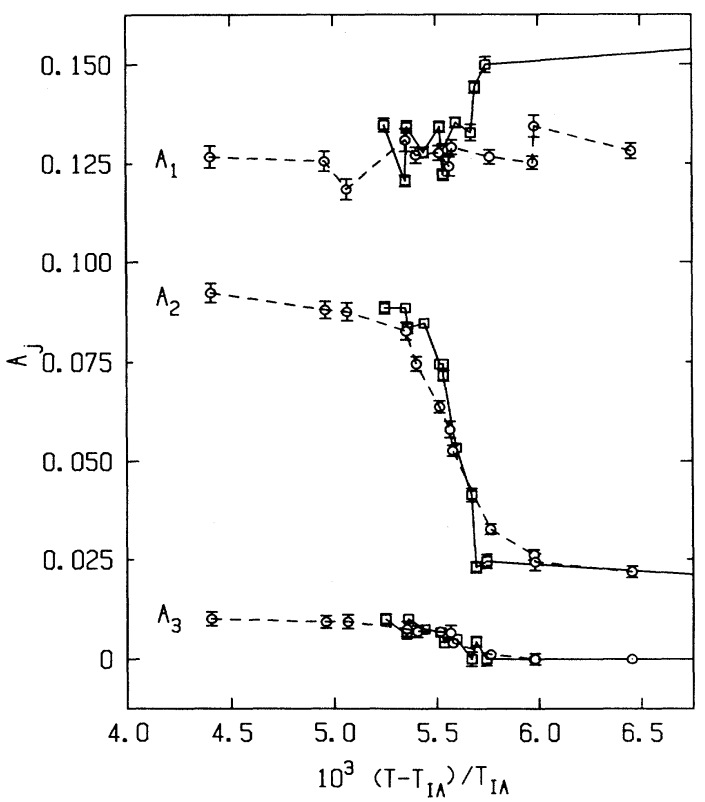

FIG. 3. Layer amplitudes for $A_{j}$ pure $12 \mathrm{CB}$ (०) and $x_{0}=0.263 \mathrm{BA}(\square)$ in $12 \mathrm{CB}$ as obtained from fits to the independent-layer model discussed in the text. The reduced transition temperatures $t_{2} \equiv\left(T_{2}-T_{I A}\right) / T_{I A}$ are not equal for pure $12 \mathrm{CB}$ and the mixture and the 12CB-BA curve has been offset by $\Delta t=7 \times 10^{-4}$ to increase the clarity of the comparison. The "sharpening" of $j=1 \rightarrow j=2$ is clearly evident. The fact that amplitudes $A_{1}$ and $A_{3}$ show very little change in the temperature region of $n=2$ formation suggests that a Landau theory ignoring explicit coupling of $A_{2}$ to these parameters may be applicable. The lines are guides to the eye. 
cause neither $R\left(0.15 \AA^{-1}, T\right)$ nor $T_{I A}$ stabilized over the course of several days.

For the mixtures listed, the curves $R\left(0.15 \AA^{-1}, T\right)$ were measured and bulk transition temperatures were measured. Also measured were a number of specular reflectivities, one at a temperature below $T_{2}$ and one above $T_{2}$ for most samples; for the $x_{0}=0.145$ sample, reflectivities were measured at 12 temperatures in the vicinity of $j=1 \rightarrow j=2$. The reflectivities could be fitted with curves generated by the electron-density model Eq. (6) with parameters that did not significantly differ from those used for pure 12CB; these reflectivities will be discussed in Sec. V. Two significant effects are apparent from the $R\left(0.15 \AA^{-1}, T\right)$ data alone. First, there is a dramatic suppression of the bulk $\left(T_{I A}\right)$ and surface $\left(T_{j}\right)$ transition temperatures; this is shown for $T_{I A}$ and $T_{2}$ in Fig. 4. Second, there is an apparent "sharpening" of the transitions $j \rightarrow j+1$. This is quite dramatic for the larger concentrations, as can be seen in Fig. 5, where $R\left(0.15 \AA^{-1}, t\right)$ is plotted for pure $12 \mathrm{CB}$ and $x_{0}=0.263$ $\mathrm{BA}$ in $12 \mathrm{CB}$. This sharpening increases as concentration is increased to $x_{0}=0.145$; for higher concentrations, the shape of $R\left(0.15 \AA^{-1}, T\right)$ remains virtually unchanged and could be described as a step function with some tempera-

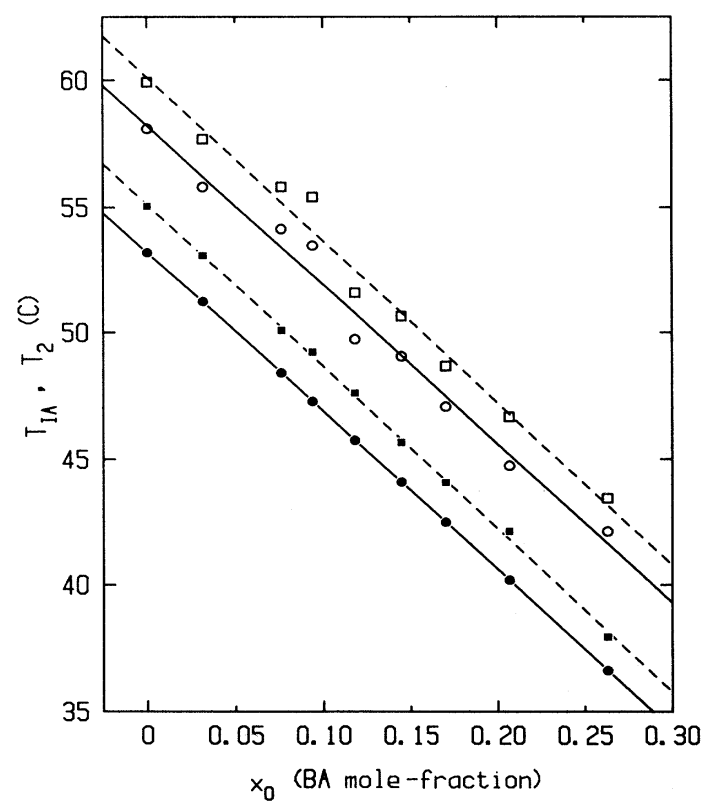

FIG. 4. Bulk $I$ - $A$ transition temperatures $T_{I A}(\circ)$ and second-layer transition temperature $T_{2}(\square)$ for nine concentrations of $\mathrm{BA}$ in $12 \mathrm{CB}$, shown with linear fits in the upper part of the figure. The fits have a slope of $d T_{I A} / d x=$ $-64 \pm 3.0 \mathrm{~K} /$ mole fraction. The scatter in the points is presumably due to systematic errors in determining the concentrations, since solid squares indicate where the $T_{2}$ would appear if the concentrations were adjusted to such that data for $T_{I A}$ fall on the solid line. (Both $T_{I A}$ and $T_{2}$ for this adjustment have been shifted for clarity.) The difference $T_{2}-T_{I A}$ is nearly constant, $T_{2}-T_{I A} \approx 1.77 \mathrm{~K}$.
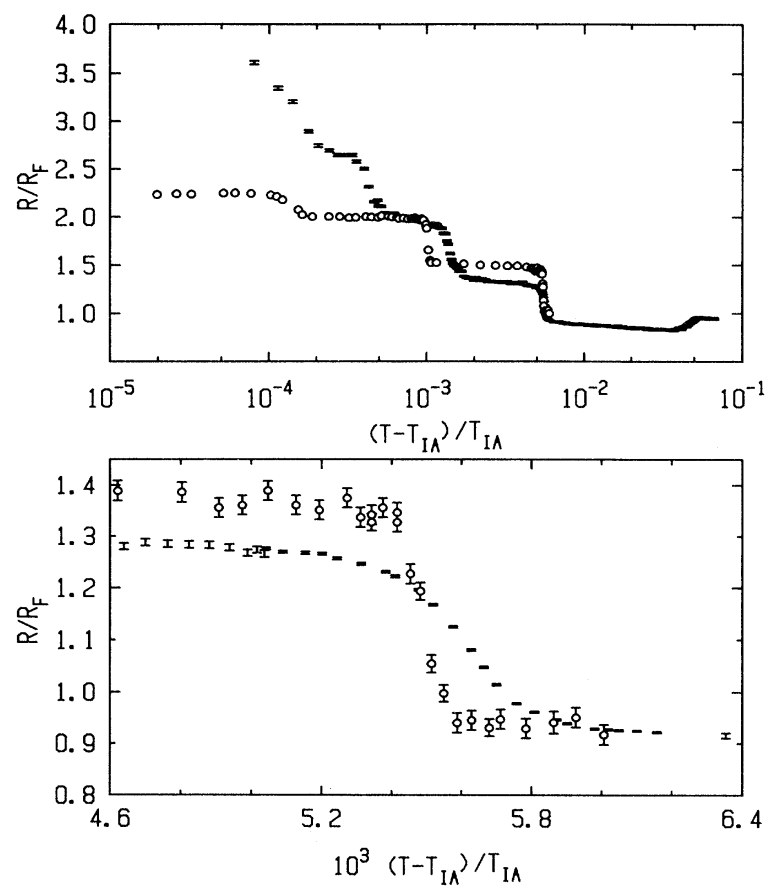

FIG. 5. Reflectivity $R\left(0.15 \AA^{-1}, t\right)$ at fixed $q_{z}=0.15 \AA^{-1}$ for pure $12 \mathrm{CB}(\cdot)$ and $x_{0}=0.263 \mathrm{BA}$ in $12 \mathrm{CB}(\circ)$.

ture smearing, as well as small pre- and post-transitional tails.

The surface transition temperatures $T_{j}$ were determined by fitting $R\left(0.15 \AA^{-1}, T\right)$ in the region of the transition to a form consisting of an error function centered at $T_{j}$, with additional constant and linear background terms. Though we do not expect this specific form to have any physical significance, the fitted values of $T_{j}$ should be relatively accurate.

Table I lists $T_{I A}$ and $T_{j}$ as a function of $x_{0}$. The $T_{I A}$ results were reproduced by observing the onset of birefringence for samples in sealed glass capillaries submerged in a water bath with $\pm 0.1^{\circ} \mathrm{C}$ temperature control. The transition temperatures measured in this way were found to be stable to within $\pm 0.1^{\circ} \mathrm{C}$ after an short initial period of drift, and no evidence of demixing was observed for periods of up to two weeks.

While there is some scatter in the data of Fig. 4, the suppression of both $T_{I A}$ and $T_{2}$ is primarily linear in $x_{0}$. A linear fit for $T_{I A}$ gives $d T_{I A} / d x_{0}=-63.9 \pm 2.7 \mathrm{~K} /$ mole fraction, and for $T_{2} d T_{2} / d x_{0}=-64.3 \pm 2.8$. The residuals for $T_{I A}, \Delta T=T_{f i t}-T_{I A}$, are large for $x_{0}=0.076(\Delta T=$ $0.74 \mathrm{~K}), x_{0}=0.09(\Delta T=1.20 \mathrm{~K})$, and $x_{0}=0.118$ $(\Delta T=-1.00 \mathrm{~K})$, but $|\Delta T| \leq 0.55 \mathrm{~K}$ for the remainder. We attribute the scatter primarily to uncertainties in the initial concentration $x_{0}$, but also to temperature drifts. As $R\left(0.15 \AA^{-1}, T\right)$ was measured repeatedly for a sample, $T_{2}$ was observed to drift; after an initial drift of $d\left(T_{2}\right) / d t \sim-10$ to $-20 \mathrm{mK} / \mathrm{h}$, a steady-state drift of $\left|d\left(T_{2}\right) / d t\right| \leq 2 \mathrm{mK} / \mathrm{h}$ would obtain after 6-12 h. Because the drift was usually positive, we attribute this to loss 
of BA, possibly through transport through the vapor to the inner surfaces of the cell, loss of vapor through poor O-ring seals, or slow phase separation in which a BApoor region developed at the surface. In any case, the measurements for $T_{2}$ quoted are those corresponding to a measurement of $T_{I A}$ which immediately followed, to minimize the intervening drift. The bulk transition temperature was usually measured only once, at the end of the reflectivity measurements, because it became clear that the appearance of a significant amount of bulk smectic led to phase separation, as evidenced by a large positive shift in $T_{2}$ and $T_{I A}$ upon reheating.

While the effects of drifts or uncertainty in $x_{0}$ introduce significant error in the values of $T_{I A}$ and $T_{2}$, their relative values appear to have much less sampleto-sample variation, as we would expect. The difference $T_{2}-T_{I A}$ is nearly constant, with an average value $T_{2}-T_{I A}=1.77 \mathrm{~K}$. Though the scatter in $T_{2}-T_{I A}$ is smaller than that in linear fits to either of these temperatures as a function of $x_{0}$, it is still large, with a standard deviation of $\sigma_{T} \approx 0.13 \mathrm{~K}$. This is significantly larger than what one would expect from the uncertainties in the measurements of $T_{I A}$ and $T_{2}$, the combined errors from which, even in the presence of (corrected) drifts, should

TABLE I. 12CB-BA bulk transition and layer transition temperatures. The bulk transition temperatures $T_{I A}$ for concentrations $x_{0}$ of $\mathrm{BA}$ in $12 \mathrm{CB}$ are listed with the layering temperatures $T_{i}$ and reduced temperatures of layering $t_{i}$ for layers $1-5$ (where measured). The true reduced temperatures are $10^{-3} \times$ the values shown, e.g., $4.7 \rightarrow 4.7 \times 10^{-3}$. The uncertainty in the values shown is $\Delta T \approx \pm 0.005^{\circ} \mathrm{C}$; in reduced temperature, $\Delta t \approx \pm 10^{-4}$.

\begin{tabular}{lllllll}
\hline \hline$x_{0}$ & $T_{I A}$ & $T_{1} / t_{1}$ & $T_{2} / t_{2}$ & $T_{3} / t_{3}$ & $T_{4} / t_{4}$ & $T_{5} / t_{5}$ \\
\hline 0 & 58.086 & 73.7 & 59.930 & 58.533 & 58.266 & 58.122 \\
& & 47 & 5.57 & 1.35 & 0.42 & 0.011 \\
0.031 & 55.815 & & 57.672 & 56.258 & 55.926 & 55.857 \\
& & & 5.64 & 1.36 & 0.33 & 0.013 \\
0.076 & 54.146 & & 55.810 & & & \\
& & & 5.08 & & & \\
0.090 & 53.490 & & 55.418 & 53.930 & 53.594 & 53.5 \\
& & & 5.90 & 1.35 & 0.32 & 0.003 \\
0.118 & 49.735 & & 51.579 & & & \\
& & & 5.71 & & & \\
0.145 & \multirow{4}{*}{49.057} & & 50.637 & & & \\
& & & 4.90 & & & \\
0.170 & 47.086 & & 48.672 & 47.419 & 47.166 & \\
& & & 4.95 & 1.04 & 0.25 & \\
0.207 & 44.747 & & 46.676 & 45.153 & 44.833 & \\
& & 6.07 & 1.28 & 0.43 & \\
0.263 & 41.710 & & 43.439 & 42.028 & 41.761 & \\
& & 5.49 & 1.01 & 0.16 & \\
\hline \hline
\end{tabular}

be $\Delta T \leq 0.02 \mathrm{~K}$. The origin of this scatter is uncertain, but one possibility is a difference between the local concentrations in the surface region, where $T_{2}$ was measured, and the bulk, where $T_{I A}$ is determined. This difference may vary randomly from sample to sample. Because of the large fluctuations in $T_{2}-T_{I A}$, any small trends would be difficult to determine.

The linear dependence shows that 12CB and BA follow Raoult's law for ideal solutions [38]. This is also in accordance with the Landau theory coupling $\psi$ and $x_{0}$ which we present below.

All samples with concentrations up to $x_{0}=0.145$ were found to have a homogeneous surface phase at temperatures in the vicinity of the $j=1 \rightarrow j=2$ transition. This was determined by translating the sample through the beam at $q_{z}=0.15 \AA^{-1}$ with narrow vertical incident slits, so that a small $\mathrm{x}$-ray footprint traversed the sample. These scans yielded flat intensities over the sample, even at temperatures in the center of the transition; we conclude from this that all parts of the sample surface possessed the same structure at a given temperature. Scans of the detector vertically through the reflected beam were also performed with a small x-ray footprint located at five positions along the length of the sample. These scans showed no broadening relative to scans taken at high temperatures or in the plateau regions where there was very little dependence of reflectivity on temperature; the reflected beam was also observed to be centered at the correct position for reflection from a horizontal surface. The combination of sample and detector scans shows that the surface was macroscopically flat over almost $70 \mathrm{~mm}$ for most samples.

Sample scans for $x_{0} \geq 0.170$ were flat in the plateau regions, but showed characteristic shapes in the vicinity of the layering transitions. Figure 6 shows six scans for $x_{0}=0.263(10 \%)$ in the vicinity of the the $j=1 \rightarrow j=2$ transition with an x-ray footprint of $2.7 \mathrm{~mm}$ along the beam direction and $5 \mathrm{~mm}$ in the transverse direction. These and other scans at interleaving temperatures show a smooth evolution from flat scans to nonuniform scans in the region of the transition, and finally flat scans at the other side of the transition. As with the lower concentrations, detector height scans taken with the $\mathrm{x}$-ray spot on different parts of the sample in the center of the transition showed no broadening relative to scans in the plateaus, and the reflected beam was in the correct position for specular reflection from a flat surface. This indicates that the observed intensity variation is not the effect of macroscopic distortions of the surface (figure error). From quantitative analysis of the data represented in the figure it is clear that, over the most of the sample, the reflected intensity could be described as $R\left(0.15 \AA^{-1}, T\right)_{X}=R\left(0.15 \AA^{-1}, T-T_{e f f}(X)\right)$, where $X$ is the longitudinal position of the $\mathrm{x}$-ray spot and $T-T_{\text {eff }}(X)$ appears to vary by a few tenths of a degree across the sample. It is also clear that $d T_{\text {eff }}(X) / d X$ is approximately linear across most of the sample. From the slope of the linear region in the $T=43.182^{\circ} \mathrm{C}$ curve of Fig. 6, this can be estimated as $\left|d T_{\text {eff }}(X) / d X\right| \approx 1.4$ $\mathrm{mK} / \mathrm{mm}$. 


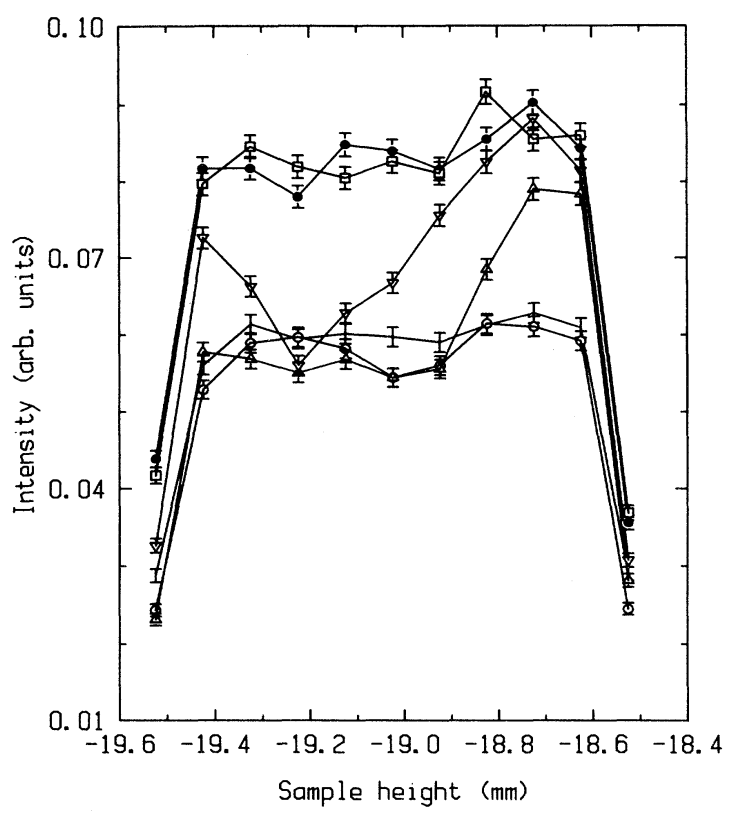

FIG. 6. Intensity reflected from the surface of a $12 \mathrm{CB}$ sample, with $x_{0}=0.263 \mathrm{BA}$, as the sample is translated through the incident beam. Shown are reflectivities at eight temperatures in the vicinity of $j=1 \rightarrow j=2: T=43.42^{\circ} \mathrm{C}$ $(\cdot), 43.352{ }^{\circ} \mathrm{C}(\circ), 43.246{ }^{\circ} \mathrm{C}(\triangle), 43.182^{\circ} \mathrm{C}(\nabla), 43.112^{\circ} \mathrm{C}$ $(\bullet), 43.078^{\circ} \mathrm{C}(\square)$. The shape of the scans in the center of the transition shows that the local layering transition temperature varies across the surface of the sample.

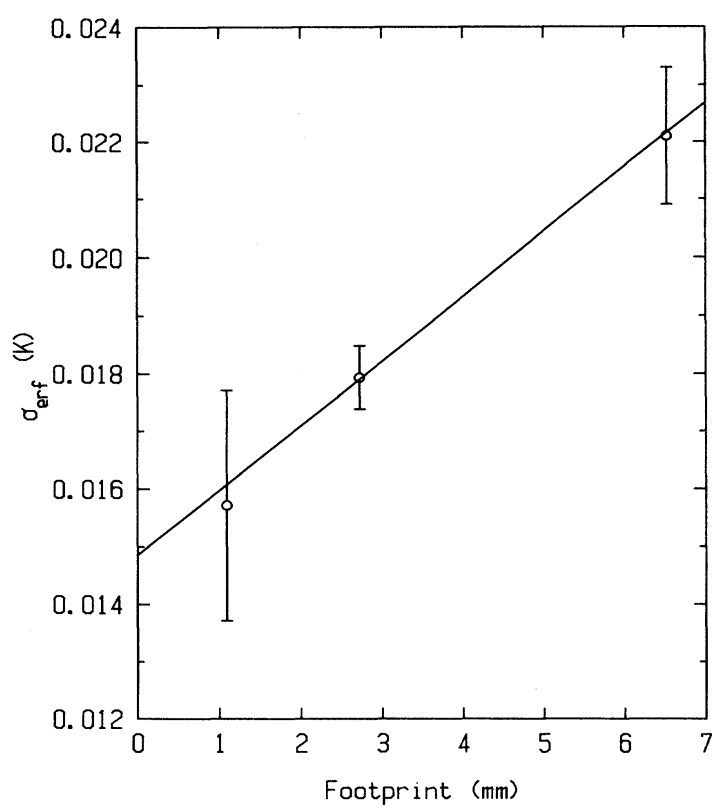

FIG. 7. The widths of error-function fits to temperature scans as a function of the $\mathrm{x}$-ray footprint size on the sample of $x=0.263 \mathrm{BA}$ in $12 \mathrm{CB}$. The slope is equivalent to a temperature gradient of $1.118 \mathrm{mK} / \mathrm{mm}$; the fact that the intercept is at positive width $\sigma_{\text {erf }}$ shows that either the transition has an intrinsic width of $\sigma_{\text {erf }}=0.0149 \mathrm{~K}$ or that other inhomogeneities - on small length scales or transverse to the beam-broaden a sharper transition.
The temperature dependence and position dependence of the reflectivity suggests that a true first-order transition could be taking place, but at different temperatures along the surface, due to temperature or composition gradients, as expressed by $R\left(0.15 \AA^{-1}, T-T_{\text {eff }}(X)\right)$. This would lead to a measured nonzero width of the transition, due to the finite portion of the sample illuminated by $\mathrm{x}$ rays. To explore this, temperature scans were performed with x-ray footprints of $6.5 \mathrm{~mm}, 2.7 \mathrm{~mm}$, and $1.1 \mathrm{~mm}$, and an error function was fitted to the step in $R\left(0.15 \AA^{-1}, T\right)$ corresponding to $j=1 \rightarrow j=2$. The fitted error-function widths of these scans are displayed in Fig. 7. The linear fit is reasonable, and has an intercept of $\sigma_{\text {erf }}=0.015= \pm 0.003 \mathrm{~K}$. The slope is $\left|d \sigma_{\text {erf }} / d X\right|=1.1$ $\mathrm{mK} / \mathrm{mm}$, very close to the estimate for $\left|d T_{\text {eff }}(X) / d X\right|$ above.

The residual width of $0.015 \mathrm{~K}$ could express the fact that the transition is not first order, or that the same apparent temperature or composition gradients evidenced by the sample scans are also present in the lateral direction, where the intensity is averaged by the $5 \mathrm{~mm}$ wide footprint.

What is the source of the apparent gradient in $T_{2}(X)$ ? Temperature gradients are unlikely, as it was previously verified through measurements with calibrated thermistors that temperature gradients from the center to the edge of the sample were $|\Delta T| \leq 0.005 \mathrm{~K}$, whereas the value of $d T_{2} / d X$ above would lead to a $0.07-0.1 \mathrm{~K}$ temperature gradient across the entire sample. On the other hand, a concentration gradient of $d x_{0} / d X=(1.8-2.2)$ $\times 10^{-5} \mathrm{~mm}^{-1}$ would have the same effect as a temperature gradient. At present we do not understand the origin of this behavior for higher concentration samples.

In summary, we have found that the addition of BA to $12 \mathrm{CB}$ reduces both the bulk transition temperature $T_{I A}$ and surface transitions temperatures $T_{j}$ nearly linearly with concentration; BA "sharpens" the layering transitions evidenced in $R\left(0.15 \AA^{-1}, T\right)$; and that we can make good fits to the data using a model where the only significantly changing parameter is $A_{2}$, the amplitude of the second layer. In particular, while the thickness $d$ does change as a function of temperature to well outside the fitted error bars, the relative change of $A_{2}$ across the transition is much larger - an increase of over a factor of 4 as temperature is lowered. To a first approximation the change in the full reflectivity, and in the constant- $q_{z}$ $R\left(0.15 \AA^{-1}, T\right)$, can be understood to be an effect of $A_{2}$ alone. This last observation will be used in the Landau theory analysis of Sec. V.

\section{B. 10CB and 10CB-BA mixtures}

For pure 10CB, two samples were measured, with bulk transition temperatures of $T_{I A}=50.429 \pm 0.005^{\circ} \mathrm{C}$ and $T_{I A}=49.975 \pm 0.005^{\circ} \mathrm{C}$. A single concentration of BA in $10 \mathrm{CB}, x_{0}=0.274$, was measured in two samples as well, with bulk transition temperatures of $T_{I A}=32.278 \pm$ $0.005^{\circ} \mathrm{C}$ and $T_{I A}=32.349 \pm 0.005^{\circ} \mathrm{C}$.

Specular reflectivities for pure $10 \mathrm{CB}$ were measured at 14 reduced temperatures ranging from $t=1.19 \times 10^{-3}$ to 
$t=2.144 \times 10^{-2}$; nine reflectivities for the first sample and five for the second. For the mixtures, specular reflectivities were measured for 21 temperatures in the range $t=1.9 \times 10^{-4}$ to $t=1.4 \times 10^{-1} ; 17$ for the first sample and four for the second sample.

In both cases, these concentrated on the region of the $j=1 \rightarrow j=2$ transition, as determined through model fits. The form of the specular reflectivities is quite similar to that for pure $12 \mathrm{CB}$ in Fig. 2, but with the primary maximum shifted to larger $q_{z}$, due to the smaller layer spacing in 10CB. Also, because the number of layers at the lowest temperatures was significantly greater than in $12 \mathrm{CB}$ (10 for pure $10 \mathrm{CB}, 5-6$ for pure $12 \mathrm{CB}$ ), this maximum showed a greater intensity than in pure $12 \mathrm{CB}$ at the lowest temperatures. Data for temperatures significantly below $j=1 \rightarrow j=2$ will not be discussed here.

Figure 8 shows the curves $R\left(q_{z}^{*}=0.1675 \AA^{-1}, t\right)$ for $10 \mathrm{CB}$ and 10CB-BA. Both curves are significantly different than the measurements $R\left(0.15 \AA^{-1}, t\right)$ in $12 \mathrm{CB}$ and $12 \mathrm{CB}-\mathrm{BA}$. First, for both the changes in intensity which were found to signal the addition of layers occur at significantly higher reduced temperature than for the longer molecule. Second, the changes in intensity are far less localized than for the 12CB samples: gone are the steplike changes which signal the discrete addition of layers, replaced in the case of pure 10CB by a gradual increase in intensity (for $j>1$ ) upon which are superimposed small variations that can be identified with layer growth. There are two immediately apparent similarities with the $12 \mathrm{CB}$ and 12CB-BA cases, however: a significant suppression of bulk and surface transition temperatures which leaves

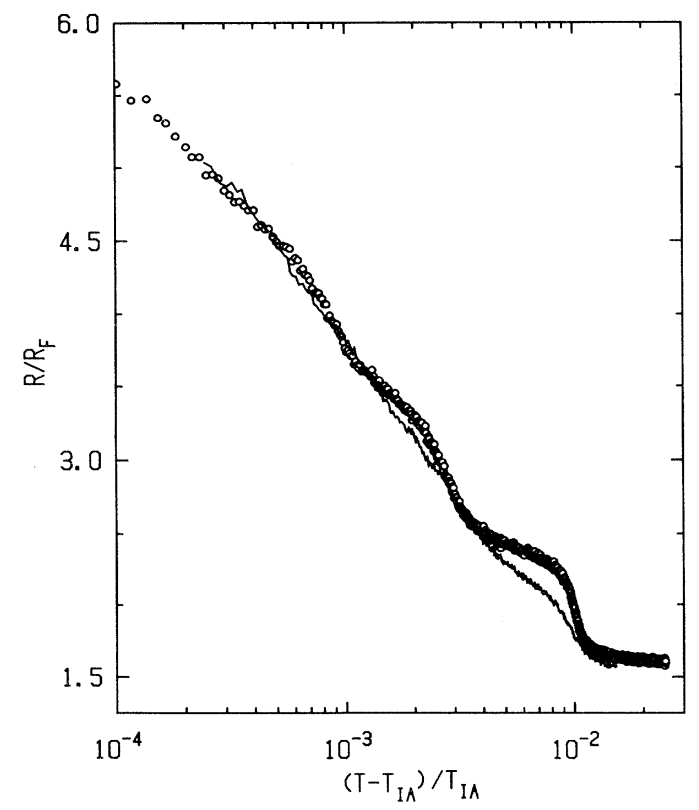

FIG. 8. Normalized reflectivity $R\left(0.1675 \AA^{-1}, t\right)$ for pure $10 \mathrm{CB}(\cdot)$ and $x_{0}=0.174 \mathrm{BA}$ in $10 \mathrm{CB}(\circ)$. The dramatic "sharpening" of the apparent transitions illustrates an effect analagous to that of $\mathrm{BA}$ in $12 \mathrm{CB}$. the location of features in $R\left(0.1675 \AA^{-1}, t\right)$ virtually unchanged accompanied by a "sharpening" of those features associated with layer growth.

From the appearance of $R\left(0.1675 \AA^{-1}, T\right)$, it would be expected that the temperature dependence of surface smectic order is significantly different than that for 12CB and $12 \mathrm{CB}-\mathrm{BA}$. This was verified by fitting the reflectivities using the model electron density Eq. (6); again, the only significantly temperature-dependent parameters are the layer amplitudes $A_{j}$.

As with $12 \mathrm{CB}$, the only other model parameter showing significant variation with $t$ is the layer spacing $d$. There is a large change in $d$, primarily across the temperature range which the fits associate with $j=2$ growth $\left(7 \times 10^{-3} \leq t \leq 2 \times 10^{-2}\right)$. For pure $10 \mathrm{CB}$ at the highest temperatures, the thickness is $\bar{d}=32.18 \pm 0.12 \AA$. Below $j=2$ saturation, $d$ reaches a plateau of $\bar{d}=$ $34.58 \pm 0.01 \AA$. For $t \leq 3 \times 10^{-3}$, where further layers are growing, there is no significant change in $d$. As with 12CB, this is significantly smaller than the bulk spacing of $d=35.36 \AA$. For 10CB-BA, $d$ also shows some temperature dependence, increasing from $d \approx 31 \AA$ to $d \approx 34.5 \AA$ as temperature was lowered through $j=1 \rightarrow j=2$.

For pure $10 \mathrm{CB}$, the position of the center of the first

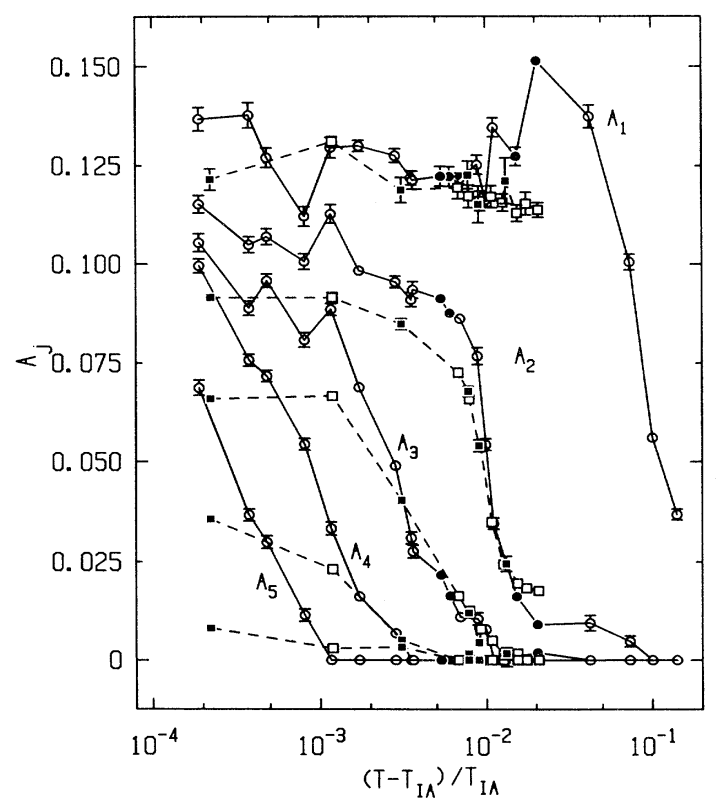

FIG. 9. Layer amplitudes for pure 10CB ( $\square$, sample 1, $\square$, sample 2) and $x_{0}=0.247 \mathrm{BA}$ in 10CB (०, sample 1 , -, sample 2) as obtained from fits to the independent-layer model. Amplitude $A_{1}$ is nonzero for all temperatures, even as high as $30 \mathrm{~K}$ above $T_{I A}$. Though $A_{1}$ shows some variation in the temperature region over which $A_{2}$ changes for both pure 10CB and 10CB-BA, it is essentially constant below $t \approx 5 \times 10^{-2}$. Only $A_{3}$ shows appreciable variation before $A_{2}$ saturates. Amplitudes $A_{3}, A_{4}$, and $A_{5}$ show a substantial region of simultaneous growth. 
bilayer, $z_{0}$, was nearly constant for each sample, though there was a significant sample-to-sample difference. For sample 1 , the average is $\overline{z_{0}}=18.05 \pm 0.03 \AA$, while for sample 2 it is $\overline{z_{0}}=17.13 \pm 0.04 \AA$. The larger value for sample 1 could be due to the presence of surface contamination, though the difference in $z_{0}$ is somewhat smaller than what one would expect for even a small organic contaminant (at least $2-3 \AA$ ). If due to contamination, this difference shows that impurities which are surface specific do not significantly affect the layering behavior.

The surface roughness $\sigma_{s}$ as a function of $t$ shows a gradual change from $\sigma_{s}=4.54 \pm 0.04 \AA$ to $\sigma_{s}=4.82 \pm$ $0.05 \AA$ as temperature is reduced.

For 10CB-BA, there is a great deal of variation in $z_{0}$ which is not attributable to the different samples; this is true of $\sigma_{s}$ as well. The consistency of $A_{2}$ and $d$ values suggests that the variations in $z_{0}$ and $\sigma_{s}$ may be attributable to systematic errors or inadequacies of the model; these deficiencies do not seem to reduce the model's sensitivity to the the layer spacing or amplitudes, which show remarkable consistency between samples.

Figure 9 shows the fitted layer amplitudes $A_{1}-A_{5}$ as a function of reduced temperature for 10CB and 10CB-BA. As expected, the changes in slope of $R\left(0.1675 \AA^{-1}, t\right)$ are accompanied by changes in $A_{3}$ and $A_{4}$ as well. Layer 1 is seen to be essentially constant below $t=5 \times 10^{-2}$. Amplitude $A_{2}$ is the only amplitude which grows to near saturation without appreciable simultaneous growth of other layers. Even while $A_{2}$ is changing significantly, there is some growth of $A_{3}$ and $A_{4}$. As indicated in the figure, the differences between samples of the same composition are on the order of the fitted error bars.

\section{LANDAU THEORY FOR $j=1 \rightarrow j=2$}

The simplest applications of Landau theory are not expected to be able to produce the incomplete smectic wetting phase diagram of long-chain liquid crystals because there is no clear way for such continuum theories to produce the discrete, well-separated layering transitions experimentally observed [21]. However, the conceptual and computational simplicity of Landau theory, and the lack of compelling agreement between experiment and other existing mean field theories [21-25], argue in favor of its use.

Furthermore, by focusing on the growth of a single layer, the difficulty of describing discrete layer formation is avoided. As shown above, in the vicinity of $j=1 \rightarrow$ $j=2$ in $12 \mathrm{CB}$, the topmost layer is saturated, while there is virtually no order in the third layer. For this restricted problem, the remainder of the material may be ignored.

The free energy which must be minimized is taken to be of Landau-de Gennes form [39], with an additional surface term of the form $F_{s} \sim-h(z)|\psi(\mathbf{r})|$, where $h(z)$ is a surface field and $F_{s}$ is taken to be negative to promote surface order. Since the problem is to be solved in mean field approximation, and we assume that there is no dependence on coordinates parallel to the interface, we can write $\psi(\mathbf{r})=\psi(z)$. A further approximation is to discretize the $z$ axis, so that the order parameter becomes $\psi(z)=\psi_{1}\left(z_{1}\right), \psi_{2}\left(z_{2}\right), \ldots$, where $z_{i}$ is the position of the $i$ th layer. This approximation appears to be valid for $12 \mathrm{CB}$ and $12 \mathrm{CB}-\mathrm{BA}$, where the $\psi_{1} \approx$ const and $\psi_{3} \approx 0$ in the temperature range of interest. Using this approximation and taking $\psi(z)$ real, the free-energy expansion for the second layer becomes

$$
\begin{aligned}
F\left(P, T, \psi_{2}\right) & -F(P, T, 0) \\
= & \frac{1}{2} A_{0}\left(T-T_{c}\right) \psi_{2}^{2}+\frac{B}{4} \psi_{2}^{4}+\frac{C}{6} \psi_{2}^{6}-h \psi_{2} .
\end{aligned}
$$

The lowest order temperature dependence has been included in the coefficient of the $\psi_{2}^{2}$ term, $A(T) \equiv A_{0}(T-$ $\left.T_{C}\right)$.

In the case of a mixture, the free energy must be expressed in terms of two order parameters, $\psi_{2}$ and the concentration (mole fraction) of the solute, $x=$ $n_{s o l} /\left(n_{s o l}+n_{l x}\right)$, where the $n$ 's are number densities of the solute and liquid crystal. If the region of interest is far from a phase transition in the pure solute, solutesolute interactions can be ignored [20]; this leaves us with a coupling between order parameters. The simplest allowed coupling is $F_{C} \sim x \psi_{2}^{2}$, which is positive (this yields a suppression of the transition temperature for $x>0$ ).

Additional terms arise depending on the thermodynamic ensemble used for the calculation. For a bulk transition in a puddle at constant $P$ and adjustable temperature $T, P$ and $T$ are independent variables. Assuming negligible vapor pressure for both solute and solvent, the total concentration $x$ is constant. The correct energy is then the Gibbs free energy, $G\left(P, T, \psi_{2}, x\right)$, with an entropy of mixing term.

Because a solution using the Gibbs free energy is difficult, an approximation valid for surfaces can be employed. As in the freezing of a liquid containing impurities, a region of two-phase coexistence is expected, one phase being solute rich or "unfrozen" $(I)$, the other solute poor or "frozen" (smectic $A$ ). For smectic order at the surface of a macroscopic puddle, the smectic region contains on the order of 1 part in $10^{5}$ of the total number of molecules. Approximating the bulk as an infinite reservoir of solute and solvent, the appropriate ensemble is the grand canonical ensemble, in which chemical potential, not particle number, is fixed.

The correct free energy is the grand potential, $\Omega$ :

$$
\begin{aligned}
\Delta \Omega(P, T, \psi, \mu)= & \Omega(P, T, \psi, \mu)-\Omega(P, T, 0, \mu) \\
= & \frac{1}{2} A(T) \psi^{2}+\frac{1}{4} B \psi^{4}+\frac{1}{6} C \psi^{6}-h \psi \\
& +\frac{1}{2} D x \psi^{2}+R T x(\ln x-1)-\mu_{0} x
\end{aligned}
$$

where $\mu_{0}$ is the chemical potential of the solute given an infinite reservoir of particles. Above and for the remainder of the discussion, $\psi_{2} \rightarrow \psi$.

At high temperatures in the $I$ phase $(\psi=0)$, minimization with respect to $x$ yields $\mu_{0}$, which may be substituted into Eq. (8) when $\psi \neq 0$, since the chemical potential is fixed. Minimizing Eq. (8) with respect to $x$ for $\psi \neq 0$ yields

$$
x=x_{0} e^{-D \psi^{2} / 2 R T} .
$$


Substitution into Eq. (8) gives

$\Delta \Omega=\frac{1}{2} A(T) \psi^{2}+\frac{1}{4} B \psi^{4}+\frac{1}{6} C \psi^{6}-h \psi-R T x_{0} e^{-D \psi^{2} / 2 R T}$,

and the equilibrium value of $\psi$ is given by the solution to

$$
\frac{\partial \Delta \Omega}{\partial \psi}=A(T) \psi+B \psi^{3}+C \psi^{5}-h+D x_{0} \psi e^{-D \psi^{2} / 2 R T}=0
$$

Equation (11) does not give $\psi$ in closed form; the solution must be found numerically. However, if $D \psi^{2} / 2 R T$ is small in the region of interest, the exponential can be expanded, giving

$$
\Delta \Omega=\frac{1}{2} A^{\prime}(T) \psi^{2}+\frac{1}{4} B^{\prime} \psi^{4}+\frac{1}{6} C^{\prime} \psi^{6}-h \psi-R T x_{0}
$$

The new coefficients are

$$
\begin{aligned}
A^{\prime}(T) & =A_{0}\left(T-T_{C}^{\prime}\right), \\
T_{C}^{\prime} & =T_{C}-\frac{D x_{0}}{A_{0}}, \\
B^{\prime} & =B-\frac{D^{2} x_{0}}{2 R T}, \\
C^{\prime} & =C+\frac{D^{3} x_{0}}{8 R^{2} T^{2}} .
\end{aligned}
$$

Having restored the free energy to Landau form, the usual statements regarding the role of the coefficients may now be made [40]. For $h=0$ and $B^{\prime}>0$, the transition is second order and occurs at $t=T-T_{C}^{\prime}=0$; in the immediate vicinity of the transition,

$$
\psi=\left\{\begin{array}{l}
0, \quad t>0 \\
\left|\frac{A_{0} t}{B^{\prime}}\right|^{1 / 2}, \quad t<0 .
\end{array}\right.
$$

The addition of $h>0$ results in a continuous transition without a simple power-law dependence of $\psi$ on $t$.

If $B^{\prime}=0$, the transition for $h=0$ is at the tricritical point, and $\psi \sim|t|^{1 / 4}, t<0$. Finally, for $B^{\prime}<0$, the transition becomes first order, with a jump from $\psi=0$ to $\psi \neq 0$ at a transition temperature

$$
T^{*}=T_{C}+\frac{3 B^{\prime 2}}{16 A_{0}^{\prime} C^{\prime}}
$$

Again, for $h>0$ the transition is still first order, but a numerical solution shows pretransitional growth of $\psi$ due to the influence of the external field.

There are three immediate consequences of Eqs. (9), (13), and (15). First, the transition temperature is suppressed linearly with solute concentration for a secondorder transition, and nearly linearly but with a small correction for a first-order transition; this agrees qualitatively with the experimental dependence of both $T_{I A}$ and $T_{2}$ on $x_{0}$. Second, if $B$ is positive at zero concentration, $B^{\prime}$ can be driven negative as solute is added, causing a second-order transition to become first order; in any case, the addition of impurities causes the transition to have a more first-order character. Finally, the growth of smectic order is accompanied by separation into a solute-rich $(I)$ region and a solute-poor (smectic- $A$ ) region: solute is "expelled" from the region in which smectic order grows.

The results of the independent-layer fits were used for an analysis using the full Landau theory expression Eq. (11). For this analysis, the second-layer amplitude $A_{2}(\equiv \psi)$ must be determined over a significant temperature range and with sufficient point density to clearly show relatively small changes in shape with changing concentration of solute. To measure and analyze many reflectivities is not only tedious, but introduces uncertainties due to temperature drifts and systematic errors. An alternative is to extract $A_{2}$ directly from the temperature scans. We found that a simple scaling method based on measuring $R\left(0.15 \AA^{-1}, t\right)$ and two reflectivities, one at a temperature above $j=1 \rightarrow j=2$ and one below the transition, produced results which agreed with the independent-layer analysis to within the error bars of the fitted $A_{2}$ values.

First, two full reflectivities at reduced temperatures $t_{a}, t_{b}$ were obtained and fitted to the reflectivity modeled by the electron density of Eq. (6). The temperature scan $R\left(0.15 \AA^{-1}, t\right)$ was then fitted to a form composed of an error function and a polynomial to provide values of $R$ at $t_{a}$ and $t_{b}$; any smooth fit would suffice, as long as interpolation between the experimental points in $t$ can be done. The curve $R$ was then scaled by multiplication and addition of a constant offset to yield $R_{\text {scale }}\left(t_{a}\right)=A_{2}\left(t_{a}\right)$ and $R_{\text {scale }}\left(t_{b}\right)=A_{2}\left(t_{b}\right)$. Figure 10 shows the result for pure 12CB; similar results were obtained for $x_{0}=0.145$.

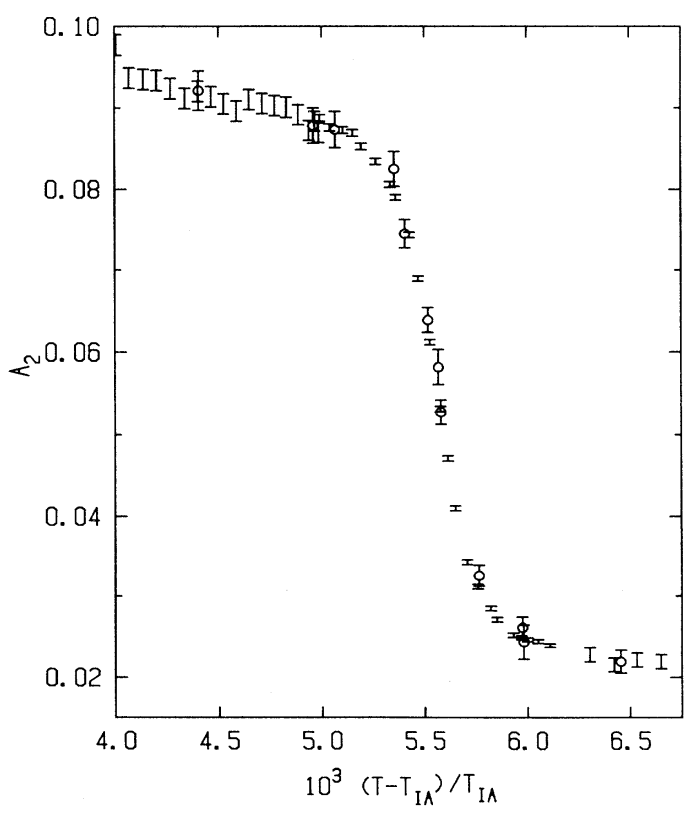

FIG. 10. Comparison between $A_{2}$ for pure $12 \mathrm{CB}$ as fitted in the independent-layer model ( $\circ$ ) and simple scaling method for obtaining $A_{2}$ from $R\left(0.15 \AA^{-1}, t\right)(\cdot)$. 
As can be seen, this method yields excellent agreement with $A_{2}$ as determined from the reflectivity fits. Assuming that this method provides a one-to-one mapping from $R \rightarrow A_{2}$, the error bars have been scaled from $R$ in the same way as $R$. The procedure was applied to all concentrations, and the resultant $A_{2}$ data used in the analysis.

Two modifications were made to the Landau theory prior to fitting. First, it was found that good fits could be obtained for temperatures below the center of the transition only if an eighth-order term $E \psi^{8} / 8$ were included in the free-energy expansion. In view of the fact that the best indication of the order of the transition is the shape in the pretransitional region on the hightemperature side, it is fortunate that the effect of the eighth-order term in this region is negligible. In addition, since the temperature dependences of all of the order parameters, for all samples with $x_{0} \geq 0.118$, appear to be essentially a step function with a common temperature smear, the data were fitted to the convolution of the Landau theory with a Gaussian of temperature width $\sigma_{T}$.

If the Landau theory is to be used to describe the effects of solutes on the $j=1 \rightarrow j=2$ layering transition, the coefficients, the coupling to concentration, the external field, and $T_{C}$ in Eq. (11) should be common to all samples. This presents some difficulty given the experimental results, in that the suppression of $T_{2}$ with increasing concentration is not smooth, but shows a good deal of scatter (see Fig. 4). Because of this scatter, the concentration $x$ was allowed to vary slightly from $x_{0}$. This appears to be justified by the rather large variation in $T_{I A}$ observed for a number of samples mixed to have the same nominal $x_{0}$ which presumably reflects uncertainties in initial concentration. Furthermore, slight changes in concentration primarily affect the transition temperature $T^{*}$ and not the shape of the transition.

In these fits, all $A_{2}$ sets were fit simultaneously with the following common parameters: coefficients $A_{0}, B, C$, and $E$; the external field strength $h / A_{0}$; the second-order coefficient coupling $A_{2}^{2}$ to $x, D / A_{0}$; the temperature at which the second-order coefficient in pure $12 \mathrm{CB}$ changes sign, $T_{C}$; and the temperature smear $\sigma_{T}$. In addition, for pure $12 \mathrm{CB}$ there were two sample-dependent parameters, a constant background $\mathcal{B}$ and a linear temperature dependence $\mathcal{L}$; the latter was included only to fit the values of $A_{2}$ at the highest temperatures, and has virtually no effect on the shape of the fitted curve in a region of \pm 0.5 $\mathrm{K}$ around the center of the transition. For samples with BA, the sample-dependent parameters were the concentration $x$, a constant background $\mathcal{B}$, and a scale factor $S$. The effect of the scale factor is to produce a fitted curve, $A_{2}^{\text {fit }}=S A_{2}^{\text {Landau }}$; if the Landau theory describes the data well, a correct choice of common parameters would yield $S=1$ for all samples.

The linear suppression of $T_{2}$ with $x_{0}$ shows that the likewise linear renormalization of $T_{C}^{\prime}$ in Eq. (13) dominates the change in transition temperature. Based on this observation, the fitted slope of $T_{2}$ vs $x_{0}$ supplies an initial guess value of $D / A_{0}=-64 \mathrm{~K} /$ mole fraction.

The data used for the Landau theory analysis are shown in Fig. 11, along with the Landau theory fits.
The curves have been offset for greater clarity, and both fits and data have been rescaled by $1 / S$ to give uniform scaling. The solid lines are the fits with temperature smear, while the broken lines show the curves without temperature smear.

The common parameters of the fits are given in Table II and the sample-dependent parameters in Table III. The relative errors in both the common parameters and the sample-dependent parameters are of the order of $1 \%$ or less; this, however, is based on the error bars produced from the temperature scans by scaling, which do not include the errors introduced by the scaling. But if we assume that the temperature scans reflect primarily the temperature dependence of $A_{2}$, the errors used for these fits should not be gross underestimates.

The important sample-dependent parameters are the fitted concentration $x$ and the scale $S$. Again, the concentration was fitted to give the correct transition temperature within the model. It was found that the fitted concentration for samples with $x_{0} \geq 0.145$ was within $5 \%$ of the nominal value, while that for lower concentrations differed from the nominal values by $15-20 \%$. This could be a result of the drifts, which were of the same magnitude for all samples: relative uncertainties in concentration due to drifts would be larger for small concentrations than for large concentrations. The only noticeable effect of small discrepancies between fitted $x$ and nominal $x_{0}$ is

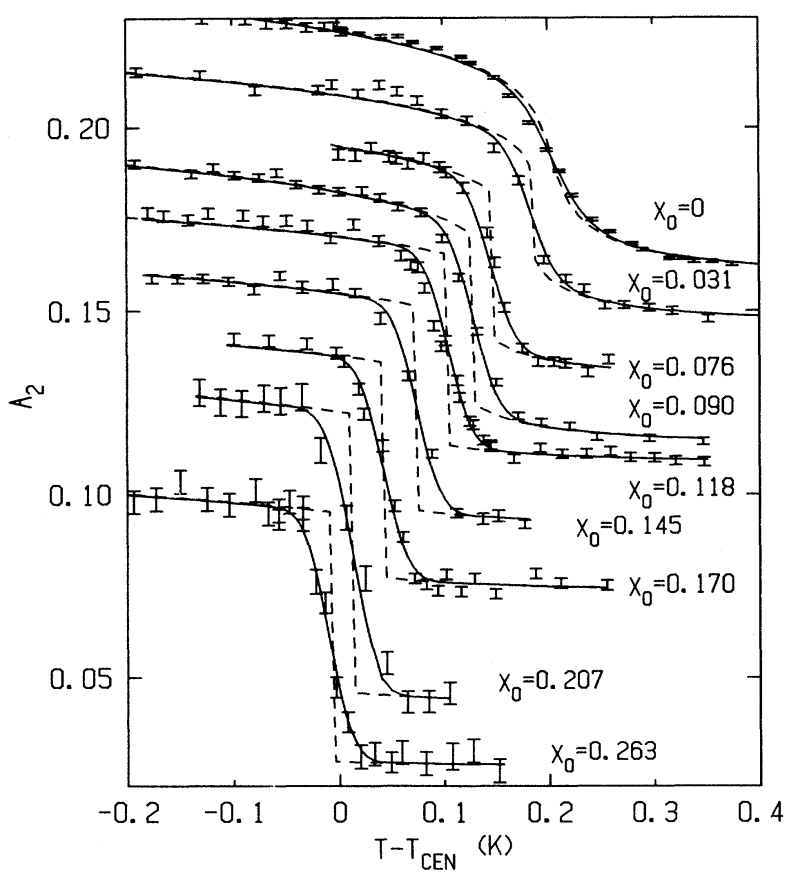

FIG. 11. Landau theory fits to the order parameter $A_{2}$ at the $j=1 \rightarrow j=2$ transition, with BA concentrations as shown. The solid lines represent the fits including a temperature smear of $\sigma_{T}=0.018 \mathrm{~K}$; the broken lines show the fitted order parameter in the absence of smearing. The fitted temperature of the center of the transition, $T_{C E N}$, has been subtracted, and the curves have been offset horizontally and vertically for clarity. 
TABLE II. Landau theory fit-common parameters. Common parameters for the Landau free-energy density used to fit the temperature dependence of $A_{2}(\psi)$. The parameters are the coefficients $A_{0}\left(\psi^{2}\right), B\left(\psi^{4}\right), C\left(\psi^{6}\right), E\left(\psi^{8}\right)$; the coupling to concentration of $\mathrm{BA}, D / A_{0}$; the surface field $h / A_{0}$; and $T_{C}$, defined by the second-order coefficient $A=A_{0}\left(T-T_{C}\right)$.

\begin{tabular}{ll}
\hline \hline$A_{0}$ & $6.98 \times 10^{5} \pm 3 \times 10^{3}$ \\
$B$ & $4.04 \times 10^{6} \pm 1.6 \times 10^{4}$ \\
$C$ & $-5.17 \times 10^{9} \pm 2 \times 10^{7}$ \\
$E$ & $2.426 \times 10^{12} \pm 6 \times 10^{9}$ \\
$D / A_{0}$ & $64.26 \pm 0.6$ \\
$h / A_{0}$ & $-8.67 \times 10^{-4} \pm 3.4 \times 10^{-6}$ \\
$T_{C}$ & $333.080 \pm 0.002$ \\
\hline \hline
\end{tabular}

a shift in the transition; shape changes sufficient to degrade the quality of the fits for this data are not created by these changes in $x$.

The average scale factor $S$ was slightly smaller than $1.0, \bar{S}=0.95$. This means that the experimentally determined magnitude of the change in order parameter across the transition is smaller than the model prediction. In principle, it is possible to change this by making the coefficient $E$ more positive, so that saturation occurs at a slightly smaller value of the order parameter. In practice, this procedure yielded poor fits. Also, the deviation of $S$ from 1.0 is not systematic; if the model overestimated the change in $A_{2}, S$ would monotonically decrease with increasing concentration. The spread in values of $S$ could be the result of systematic errors in the scaling procedure used to generate the data from the temperature scans or sample-to-sample differences not entirely attributable to the presence of BA (such as impurities on the surface). Regardless of these systematic errors, the fact that $\bar{S} \approx 1.0$ is indicative that the choice of common parameters for the fits is good.

The important results can be easily seen in Fig. 11 . As with $R\left(0.15 \AA^{-1}, T\right)$, the greatest changes in shape occur for fitted $x \leq 0.118$.

We note first that the temperature smear $\sigma_{T}$ has almost no effect on the pure 12CB fit. Furthermore, $A_{2}$ for $12 \mathrm{CB}$ with no temperature smear is continuous, showing that the transition is second order. All other concentrations without $\sigma_{T}$ show a significant jump $\Delta A_{2}$ which accounts for more than half of the total change in $A_{2}$ around the transition for concentrations as low as $x_{0}=0.076$. This results from the fact that the secondorder coefficient $B^{\prime}$ is driven strongly negative for even the $x_{0}=0.031$ sample. This means that the transition is continuous ("second order") for pure 12CB, while it is discrete ("first order") for all nonzero concentrations measured. Also apparent is the fact that the shapes of data and fits for $x_{0} \geq 0.118$ are virtually identical: preand post-transitional effects have been suppressed, and the primary effect of increasing concentration is the suppression of the temperature of the transition.

Saturation for larger concentrations is also apparent in the jump in order parameter $\Delta A_{2}$, as shown in Fig. 12. The fit parameters which yield a continuous transition for pure 12CB predict that the crossover to first-order behavior occurs at a very small concentration, $x \approx 0.016$. This would be analogous to a tricritical point in the bulk. Another possibility, to be discussed below, is that pure $12 \mathrm{CB}$ is near the critical point for $j=1 \rightarrow j=2$, and

TABLE III. Landau theory fit-individual parameters. Sample-dependent parameters used to fit the temperature dependence of $A_{2}(\psi)$ with the Landau free-energy density. For each nominal concentration $x_{0}$ of $\mathrm{BA}$, the fitted parameters are the concentration $x$; the constant background $\mathcal{B}$; and the scale factor $S$. Also given are the $\chi^{2}$ values for the individual fits.

\begin{tabular}{lllll}
\hline \hline$x_{0}$ & \multicolumn{1}{c}{$x$} & $\mathcal{B}$ & \multicolumn{1}{c}{$S$} & $\chi^{2}$ \\
\hline 0 & 0 & $0.67 \pm 0.05$ & 1 & 3.499 \\
0.0314 & $0.03576 \pm 0.00007$ & $0.2043 \pm 0.0006$ & $0.92 \pm 0.01$ & 2.849 \\
0.0761 & $0.065421 \pm 0.000001$ & $0.0220 \pm 0.0007$ & $0.93 \pm 0.01$ & 1.554 \\
0.0904 & $0.07179 \pm 0.00008$ & $0.0303 \pm 0.0006$ & $1.02 \pm 0.01$ & 2.27 \\
0.1181 & $0.132021 \pm 0.000004$ & $0.0175 \pm 0.0007$ & $0.96 \pm 0.01$ & 3.784 \\
0.1446 & $0.1469 \pm 0.0002$ & $0.0199 \pm 0.0009$ & $0.88 \pm 0.01$ & 1.705 \\
0.1702 & $0.177985 \pm 0.000003$ & $0.0176 \pm 0.0009$ & $0.89 \pm 0.02$ & 4.855 \\
0.2067 & $0.21020 \pm 0.00007$ & $0.014 \pm 0.005$ & $1.06 \pm 0.02$ & 1.888 \\
0.2631 & $0.26190 \pm 0.00006$ & $0.024 \pm 0.001$ & $0.91 \pm 0.02$ & 0.938 \\
\hline \hline
\end{tabular}




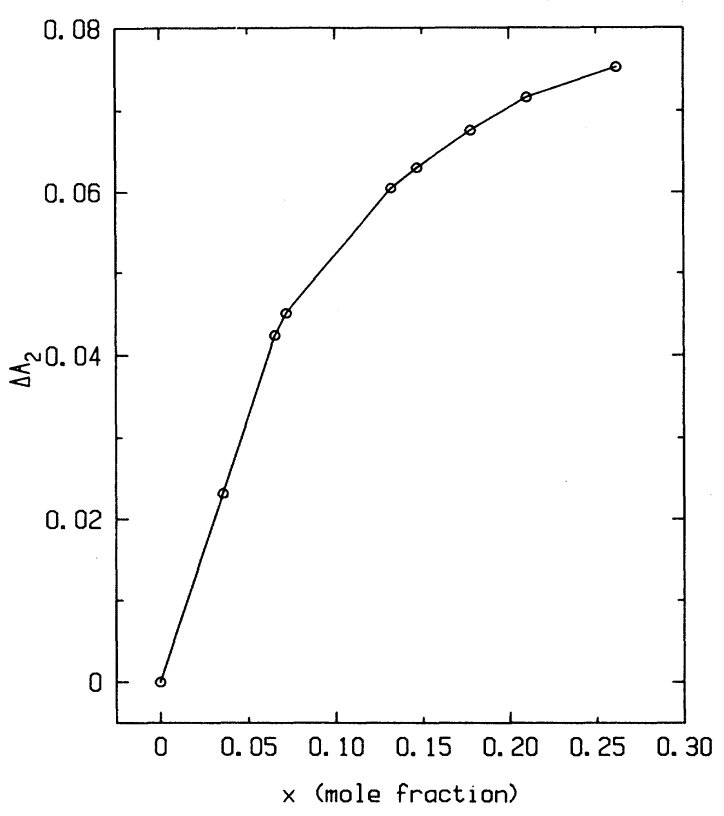

FIG. 12. Jump in order parameter $\Delta A_{2}$ at the transition as a function of fitted concentration $x$ from the best-fit Landau theory parameters. Only pure 12CB shows no discontinuity in $A_{2}$.

that the addition of impurities causes the phase diagram to shift in such a way that this critical point is crossed by scanning $T$ at constant $P$ for $x=0.016$.

The fact that $j=1 \rightarrow j=2$ appears to be continuous only in pure 12CB is cause for some suspicion that the fits have fortuitously provided a crossover such that all nonzero concentrations of BA measured are first order. Attempts were made to vary the parameters of the model to determine whether other satisfactory fits could be obtained; the details are not important, but we found that we could neither remove the temperature smear nor change the coefficients in such a way as to make all, or at least more, transitions continuous.

In summary, the best fit to the Landau theory model is one in which (a) there is a temperature smear of $0.018 \mathrm{~K}$; this is consistent with the discussion above regarding the inhomogeneous surface structure for high-concentration samples and the asymptotic value of the width of an error-function fit to $R\left(0.15 \AA^{-1}, T\right)$; (b) the transition is continuous for pure $12 \mathrm{CB}$, but discontinuous for all other samples; and (c) the effect of added impurities on the shape of the transition is negligible for $x_{0} \geq 0.118$.

\section{DISCUSSION}

What is most striking about the surface smectic structure of pure $12 \mathrm{CB}$ and 12CB-BA is not their differences, but their similarities. This can be easily seen by looking at both the reflectivities and fitted electron-density profiles for pure $12 \mathrm{CB}$ and $x_{0}=0.145 \mathrm{BA}$ in $12 \mathrm{CB}$ at temperatures above and below the $j=1 \rightarrow j=2$ transition, as shown in Fig. 13. There is only one systematic difference in the fitted parameters of the independentlayer model, other than the temperature dependence of $A_{2}$. This is that the layer spacing $d$ for the mixtures is $\approx 0.7 \AA$ smaller than that of pure $12 \mathrm{CB}$ in the $j=2$ region. Other than this, the electron density is for the most part insensitive to the presence of BA.

Where would one expect the $\mathrm{BA}$ molecules to reside in the layers? Firstly, both $12 \mathrm{CB}$ and BA have significant dipole moments, $\mu_{D}=4.95 \mathrm{D}$ for $12 \mathrm{CB}$ [41] and $\mu_{D}=5.54 \mathrm{D}$ for BA [42]. Furthermore, experiments on the homologous series $n \mathrm{CB}$ indicate that the dipole moment is primarily associated with the cyano-terminated aromatic core [41]. As a result, preferential association of BA with the core region might be expected.

Experiments addressing solute location in smectic liquid crystals are inconclusive. Moseley and Lowenstein [36] used ${ }^{13} \mathrm{C}$ NMR to study the anisotropic diffusion of methane and chloroform in a number of liquid crystals, including $7 \mathrm{CB}$ and $8 \mathrm{CB}$. They found that the diffusion constant for motion parallel to the layers in the $A$ phase was 2-5 times greater than that for perpendicular motion. By using packing and chemical affinity arguments,

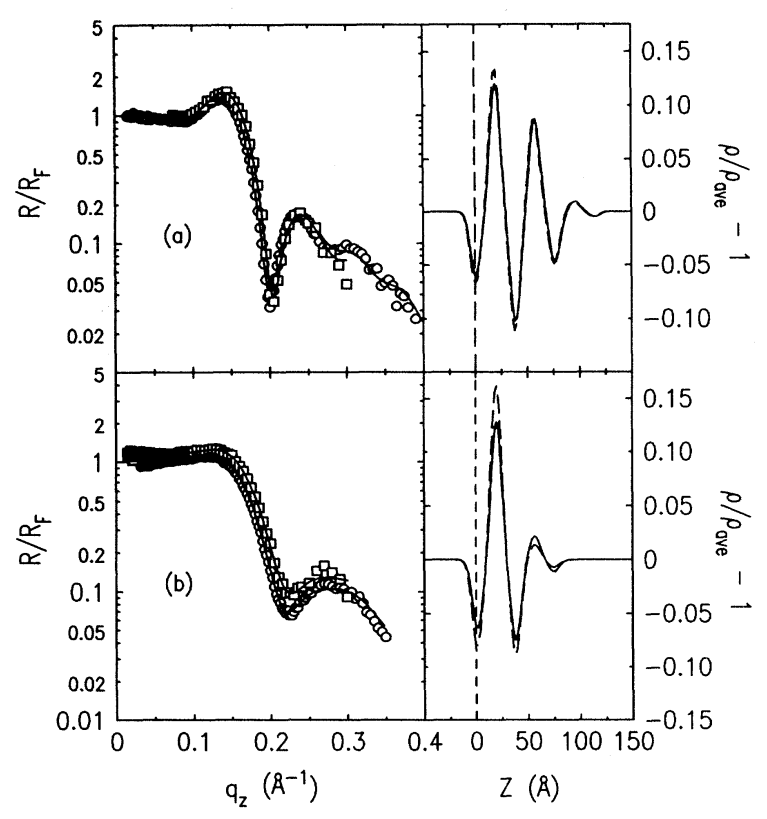

FIG. 13. Reflectivities $R / R_{F}$, and the fitted electron densities of pure 12CB (०) and $x_{0}=0.145 \mathrm{BA}(\square)$ in the $j=2$ region (a) and $j=1$ region (b). The error function used to model the liquid-vapor interface has been removed from the electron densities for clarity. The reduced temperatures of the pure $12 \mathrm{CB}$ are $t=5.07 \times 10^{-3}$ (a) and $t=6.46 \times 10^{-3}$ (b), while those for the mixture are $t=4.55 \times 10^{-3}$ (a) and $t=8.43 \times 10^{-3}(\mathrm{~b})$. Aside from the amplitude $A_{1}$ at higher temperatures, the profiles for pure $12 \mathrm{CB}$ (-) and the mixture (- ) in each region $(j=1$ or $j=2)$ are virtually identical. 
this was attributed to the expulsion of solute from the core region into the aliphatic tails, in the case of nonpolar methane, or an enhanced affinity for the core in the case of polar chloroform. In either case, motion perpendicular to the layers is impeded, while motion parallel to the layers allows the solute to remain in a region of high solubility.

On the other hand, the experiments of Guo and Fung [43] seem to indicate no preferential association. These ${ }^{13} \mathrm{C}$ NMR experiments used variable-angle spinning (VAS) and separated local field (SLF) spectroscopy to determine the nematic order parameter of different parts of the $7 \mathrm{CB}$ and $8 \mathrm{CB}$ molecules. No appreciable change in orientational order in either the cores or tails due to the addition of $x=0.08 \mathrm{CCl}_{4}$, cyclohexane, benzene, or hexane was observed. Because an affinity for one part of the liquid crystal molecule might be expected to enhance or disrupt the order of that region, they interpret their findings as evidence that preferential association does not occur.

Finally, it is possible that BA is at least partially expelled from the surface smectic layers, in analogy to bulk phase separation of a mixture. The above-cited NMR studies showed no indication of bulk phase separation in mixtures, as indicated by a lack of hysteresis; this is in contrast to the our experience with the bulk 12CB-BA transition. The difficulty we observed in remixing the samples after bulk phase separation, which is reasonable given typical diffusion constants in smectics, should not be a problem as far as phase separation in the surface region is concerned: the expulsion of $\mathrm{BA}$ as temperature is lowered, and subsequent reincorporation upon heating, could proceed rapidly because of the thinness of a surface region in contact with a bulk BA reservoir.

Experimentally, we were not able to determine any signs of phase separation in the surface region. Because the bulk electron densities for for 12CB and BA differ by only a few percent, we expect that the any differences in average electron densities between BA-depleted and BA-rich regions would be quite small. Though reflectivity is sensitive to small variations in electron density, we feel that the imperfect nature of the model used to fit the reflectivity, as well as the large number of adjustable parameters, make it unlikely that we could demonstrate such an expulsion effect for the current materials.

The most significant effect of the solute is to alter the temperature dependence of the $j=1 \rightarrow j=2$ layering transition, as well as lowering the bulk $T_{I A}$ and the individual $T_{j}$. The same qualitative effects were seen in 12CB-BA and 10CB-BA mixtures: a "sharpening" of the features in $R\left(q_{z}^{*}, T\right)$ which we have called layering "transitions" and an overall suppression of the temperatures such that $t_{j}$ are roughly the same for pure materials and mixtures.

The inhomogeneous reflectivities for $x_{0}>0.145 \mathrm{BA}$ in $12 \mathrm{CB}$ are a cause for concern, because they complicate the interpretation of the temperature dependence of the smectic amplitude $A_{2}$. However, it should be stressed that the effects are consistent from sample to sample. As discussed above, the largest possible temperature gradient across the entire sample was of the order of $|\Delta T| \leq 5$
mK. This alone would be insufficient to cause a broadening of a first-order transition by a few tenths of a degree across the surface of the sample. If the temperature gradient drives a concentration gradient, this would magnify the effect. If, on the other hand, the inhomogeneous reflectivities are the result of an incomplete initial mixing of $12 \mathrm{CB}$ and $\mathrm{BA}$, the expected diffusion of $\mathrm{BA}$ in $12 \mathrm{CB}$ would not be significant on the time scales of the experiments at the temperatures studied. In such a case, no progressive lessening of the inhomogeneities would be observable.

Sample curvature effects could also be implicated in this regard. A simple analysis [44] shows that the relative change in surface tension due to the expected curvature at the edges of the sample should be of the order of $\Delta\left|\gamma / \gamma_{0}-1\right| \approx(2-5) \times 10^{-6}$, where $\gamma_{0}$ is the surface tension at the center of the transition. A recent surface tension measurement [45] of pure 12CB shows that the relative change in surface tension over a region of about $0.25^{\circ} \mathrm{C}$ at the transition-the extent of the inhomogeneities - is $\Delta\left|\gamma / \gamma_{0}-1\right| \approx 2.5 \times 10^{-3}$. Clearly, curvature alone is not likely to be responsible for the inhomogeneities. However, it is possible that sample curvature drives a concentration gradient, in which BA is depleted from the regions near the edges of the sample.

Though the source of these inhomogeneities is unknown, inspection of the scans $R\left(0.15 \AA^{-1}, t\right)$ shows that the layering grows increasingly sharp up to $x_{0}=0.118$ BA. Even in the presence of the inhomogeneities, if a temperature broadening of $0.018 \mathrm{~K}$ is assumed, consistent with the observed position dependence of the transition on the sample surface, the transition appears to become completely first order with a concentration of $x_{0}=0.118 ;$ there is very little change in $R\left(0.15 \AA^{-1}, t\right)$ for higher concentrations.

The behavior of the second-layer amplitude $A_{2}$ is consistent with a Landau theory which was used to simultaneously fit data for nine concentrations of BA in 12CB. The observations borne out by this model include a nearly linear suppression of the transition temperature $T_{2}$; a sharpening of the transition, due to the reduction of smectic susceptibility with increasing concentration; and a larger change $\Delta A_{2}$ of the order parameter across the transition. The parameters of the Landau theory indicate that the transition in pure $12 \mathrm{CB}$ is continuous, but that the effects of the surface field and/or presence of $A_{1} \neq 0$ cause a pretransitional tail; and that the addition of as little as $1 \% \mathrm{BA}$ drives the transition first order.

While the theory has not been applied to $10 \mathrm{CB}$, it is qualitatively clear that a concentration of $x_{0}=$ $0.274(10 \%) \mathrm{BA}$ in $10 \mathrm{CB}$ still leaves a significant breadth to the transition, of the order of $\approx 1^{\circ} \mathrm{C}$. This width is significantly larger than the range of the inhomogeneities in reflectivity for $12 \mathrm{CB}-\mathrm{BA}\left(\approx 0.25^{\circ} \mathrm{C}\right)$, and is unlikely to be an artifact; furthermore, the reflectivity inhomogeneities were not observed for 10CB-BA. As a result, $x_{0}=0.274(10 \%) \mathrm{BA}$ has probably not driven the $j=1 \rightarrow j=2$ transition first order. To correctly apply the theory to 10CB would require the use of at least two order parameters, $A_{2}$ and $A_{3}$, because of the significant 
growth of $A_{3}$ in the temperature range over which $A_{2}$ grows to saturation.

The theory also predicts what one would intuitively expect, that solute should be expelled from a region as smectic order develops there. In the theory,

$$
x(\psi)=x_{0} e^{-D \psi^{2} / 2 R T} .
$$

This implies that $\approx 8 \%$ of the $\mathrm{BA}$ in the $j=2$ region of the surface is expelled when the sample goes through the $j=1 \rightarrow j=2$ transition for the highest-concentration 12CB-BA sample. As discussed above, however, the lack of scattering contrast between $12 \mathrm{CB}$ and $\mathrm{BA}$ would make the detection of such a small effect difficult.

Among other issues, future work should address this possible phase separation. The use of a solute with a greater electron density may be sufficient for this. Simulations show that the complete expulsion of a perfluorinated BA, pentafluorobenzyl alcohol (PFBA), from the surface of a mixture with bulk concentration of $x_{0}=0.24$ would cause features in the $\mathrm{x}$-ray reflectivity characteristic of the length scale over which the depletion occurs. Preliminary measurements on one sample of $x=0.24$ PFBA in 12CB were inconclusive: the data could not be fitted well with the independent-layer model as described above, but no limits on concentration differences between the surface smectic region and the isotropic bulk could be determined. This is not surprising if the expulsion reduces the concentration of solute by less than $10 \%$. Unfortunately, samples with higher concentrations of PFBA showed the same instabilities as high concentrations of BA, precluding studies that might have enhanced the hypothesized electron-density difference.

Another way of addressing this issue would be to use a solute with an atom which has an absorption edge whose wavelength is accessible at a synchrotron x-ray facility. By measuring the anomalous reflectivity with x-ray wavelengths just below and just above the absorption edge, it should be possible to reconstruct the depth profile of the atomic species of interest. A further promising possibility would be selective deuteration of solute or liquid crystal molecule for neutron reflectivity measurements. The large scattering-length contrast created in this way should allow the determination of solute concentration in the surface layers.

A further aspect of possible expulsion of material from the surface for $12 \mathrm{CB}$ has interesting implications for high-concentration mixtures, because these samples show smectic order at the surface at temperatures well below the material's bulk crystallization temperature, $T_{A K} \approx$ $48^{\circ} \mathrm{C}$. Grazing-incidence diffraction could be used to examine whether long-range order within the plane of the surface exists in the mixtures.

In some respects, the cyanobiphenyl surface-layering phase diagram resembles a generic multilayer-adsorption phase diagram [46]. As shown in previous work $[3,7]$, short-chain homologs appear to wet continuously and completely, while longer-chain molecules were originally thought to undergo a finite number of first-order layering transitions. In this analogy, short-chain molecules behave like an adsorbing gas which approaches coexistence at high temperatures, above the wetting tempera- ture $T_{W}$, while long-chain molecules behave like the gas approaching coexistence below $T_{W}$, where it forms a restricted number of solid layers. If this analogy is correct, the individual first-order layering transitions, which exist in the region of incomplete wetting, should be terminated by critical points in a phase diagram expressed in chemical potential and temperature, $(\mu, T)$; the presence of critical points has also been suggested by a recent density-functional calculation [25]. When impurities are added, we expect the phase diagram to shift such that the bulk transition occurs at a lower temperature. With this shift, transitions in which layers form continuously can change to true first-order transitions in which new layers form discretely. The result is that the experimental path in which $(\mu)$ is held fixed and $T$ varies moves from a position that does not cross a layering phase boundary (i.e., beyond a critical point) to one that cuts across a first-order layer transition line. This is suggested by the behavior of both $12 \mathrm{CB}$ and $10 \mathrm{CB}$, but especially $12 \mathrm{CB}$, where a continuous $j=1 \rightarrow j=2$ transition appears to become first order with the addition of solute. This implies that the addition of impurities has the same qualitative effect as increasing the chain length. We stress that the evidence for this analogy is merely suggestive at this point, and an exploration of this possibility should be one goal of a more comprehensive study.

A number of other observations from these experiments and our Landau theory analysis suggest directions for further study. Because the $j=1 \rightarrow j=2$ transition in 12CB appears to be marginally second order, a quantitative investigation of $j=0 \rightarrow j=1$ and $j=2 \rightarrow j=3$ should be undertaken, to determine the magnitude of sharpening for those transitions. In addition, the differences between 10CB-BA and 12CB-BA show that chain length could be a useful variable for moving along the $\mu$ axis of the surface phase diagram; in particular, measurements of 11CB are of high priority, since the $j=1 \rightarrow j=2$ transition for 10CB appears to be continuous even with large concentrations of $\mathrm{BA}$, while that for pure $12 \mathrm{CB}$ may be marginally second order and easily driven first order with small amounts of solute. While the surface inhomogeneity problem in 12CB-BA is troublesome, it should be remembered that these effects became apparent only with concentrations significantly in excess of that needed to drive the transition first order. A possible way to test the hypothesis that impurities might push the system away from complete wetting would be to use even shorter-chain homologs, which have been shown to have a larger number of surface layers at the bulk transition. Unfortunately, it was found that bulk phase separation occurs in 9CB-BA when $9 \mathrm{CB}$ is in the $N$ phase, so studies would necessarily be confined to $n>9.3$, where no $N$ phase exists. Still, these effects might be much more pronounced for these shorter molecules. Another study of merit would be of the long-chained $n$ OCB's, which in previous measurements showed layering consistent with incomplete wetting by layers added via first-order transitions [7]. Finally, as discussed above, x-ray reflectivity studies with solutes that possess heavy atoms (with large atomic number $Z$ ), or neutron reflectivity studies with deuterated solutes, could provide insight into the distri- 
bution of solute molecules within the surface layers, as well as any changes in that distribution as a function of temperature.

\section{ACKNOWLEDGMENTS}

The research described here was supported by Grants No. NSF-DMR-91-13782 and No. NSF-DMR-89-20490.
Some of the experiments were carried out on the HarvardBNL liquid surface reflectometer on beamline X22B at the National Synchrotron Light Source, Brookhaven National Laboratory. This facility is supported by DEAC02-76CH00016. M.D. gratefully acknowledges support by the U.S.-Israel Binational Science Foundation, Jerusalem.
[1] J. Als-Nielsen, F. Christensen, and P.S. Pershan, Phys. Rev. Lett. 48, 1107 (1982).

[2] P.S. Pershan and J. Als-Nielsen, Phys. Rev. Lett. 52, 759 (1984).

[3] B.M. Ocko, A. Braslau, P.S. Pershan, J. Als-Nielsen, and M. Deutsch, Phys. Rev. Lett. 57, 94 (1986).

[4] E.F. Gramsbergen, W.H. de Jeu, and J. Als-Nielsen, J. Phys. (Paris) 47, 711 (1986).

[5] P.S. Pershan, A. Braslau, A.H. Weiss, and J. Als-Nielsen, Phys. Rev. A 35, 4800 (1987).

[6] E.F. Gramsbergen and W.H. de Jeu, J. Phys. (Paris) 49, 363 (1988).

[7] Alan Braslau, Ph.D. thesis, Harvard University, 1988 (unpublished).

[8] B. Ocko, Phys. Rev. Lett. 64, 2160 (1990).

[9] P.S. Pershan, Phys. Rev E 50, 2369 (1994).

[10] P.E. Cladis, Wim van Saarloos, David A. Huse, J.S. Patel, J.W. Goodby, and P.L. Finn, Phys. Rev. Lett. 62, 1764 (1989).

[11] J. Thoen, H. Marynissen, and W. Van Dael, Phys. Rev. A 26, 2886 (1982).

[12] See W.G. Bouwman and W.H. de Jeu, Phys. Rev. Lett. 68, 800 (1992), and references therein.

[13] See G. Nounesis, M.J. Young, K.I. Blum, C.W. Garland, and R.J. Birgeneau, Z. Phys. B. 90, 357 (1993), and references therein.

[14] BDH Limited, Advanced Materials Division.

[15] J. Thoen, H. Marynissen, and W. Van Dael, Phys. Rev. Lett. 52, 204 (1984).

[16] H. Marynissen, J. Thoen, and W. Van Dael, Mol. Cryst. Liq. Cryst 97, 149 (1983).

[17] B.M. Ocko, R.J. Birgeneau, J.D. Litster, and M.E. Neubert, Phys. Rev. Lett. 52, 208 (1984).

[18] B.M. Ocko, R.J. Birgeneau, and J.D. Litster, Z. Phys. B 62, 487 (1986).

[19] M. Schick, in 1988 Les Houches Lectures, Session XLVIII, Liquids at Interfaces, edited by J. Charvolin, J. F. Joanny, and J. Zinn-Justin (Elsevier Science Publishers B.V., Amsterdam, 1990), p. 415.

[20] L.D. Landau and E.M. Lifshitz, Statistical Physics, Part 1, 3rd ed. (Pergamon, New York, 1980).

[21] Jonathon V. Selinger and David R. Nelson, Phys. Rev. A 37, 1736 (1988).

[22] Z. Pawlowska, G.F. Kventsel, and T.J. Sluckin, Phys.
Rev. A 36, 992 (1987).

[23] W.L. McMillan, Phys. Rev. A 4, 1238 (1971)

[24] L. Mederos and D.E. Sullivan, Phys. Rev A 46, 7700 (1992).

[25] A.M. Somoza, L. Mederos, and D.E. Sullivan, Phys. Rev. Lett. 72, 3674 (1994).

[26] Michael E. Fisher, J. Chem. Soc. Faraday Trans. 2 82, 1569 (1986).

[27] M. Moldover and J.W. Cahn, Science 207, 1073 (1980).

[28] M. Kahlweigt, G. Busse, D. Haase, and J. Jen, Phys. Rev. A 38, 1395 (1988).

[29] L.G. Parratt, Phys. Rev. 95, 359 (1954).

[30] A. Braslau, P.S. Pershan, G. Swislow, B.M. Ocko, and J. Als-Nielsen, Phys. Rev. A 38, 2457 (1988).

[31] D.K. Schwartz, M.L. Schlossman, E.H. Kawamoto, G.J. Kellogg, and P.S. Pershan, Phys. Rev. A 41, 5687 (1990).

[32] D. Beysens and M. Robert, J. Chem. Phys. 87, 3056 (1987).

[33] M.P. Gelfand and M.E. Fisher, Physica (Amsterdam) A 166, 1 (1990).

[34] B.M. Ocko, X.Z. Wu, E.B. Sirota, S.K. Sinha, and M. Deutsch, Phys. Rev. Lett. 72, 242 (1994).

[35] A.J. Leadbetter, J.C. Frost, J.P. Gaughan, G.W. Gray, and A. Moseley, J. Phys. (Paris) 40, 375 (1979).

[36] Michael E. Moseley and Aharon Lowenstein, Mol. Cryst. Liq. Cryst. 90, 117 (1982).

[37] A.H. Weiss, M. Deutsch, A. Braslau, B.M. Ocko, and P.S. Pershan, Rev. Sci. Instrum. 57, 2554 (1986).

[38] B. Mahan, University Chemistry (Benjamin Cummings, Menlo Park, 1987).

[39] P.G. de Gennes, Solid State Commun. 10, 753 (1972).

[40] Kerson Huang, Statistical Mechanics, 2nd ed. (John Wiley \& Sons, New York, 1987).

[41] K.P. Gueue, E. Megnassan, and A. Proutiere, Mol. Cryst. Liq. Cryst. 132, 303 (1986).

[42] C. Reichardt, Solvent Effects in Organic Chemistry (Verlag-Chemie, New York, 1979).

[43] Wen Guo and B.M. Fung, Liq. Cryst. 9, 117 (1991).

[44] Arthur W. Adamson, Physical Chemistry of Surfaces, 5th ed. (John Wiley \& Sons, New York, 1990).

[45] X.Z. Wu (private communication).

[46] R. Pandit, M. Schick, and M. Wortis, Phys. Rev. B 26, 5112 (1982). 